\title{
The Undecidability of Boolean BI through Phase Semantics (full version)
}

\author{
Dominique Larchey-Wendling ${ }^{\dagger}$ and Didier Galmiche ${ }^{b}$ \\ LORIA - CNRS $^{\dagger}$ - UHP Nancy ${ }^{b}$ UMR 7503 \\ BP 239, 54506 Vandœuvre-lès-Nancy, France \\ \{larchey, galmiche\}aloria.fr
}

\begin{abstract}
We solve the open problem of the decidability of Boolean BI logic (BBI), which can be considered as the core of separation and spatial logics. For this, we define a complete phase semantics for BBI and characterize it as trivial phase semantics. We deduce an embedding between trivial phase semantics for intuitionistic linear logic (ILL) and Kripke semantics for BBI. We single out a fragment of ILL which is both undecidable and complete for trivial phase semantics. Therefore, we obtain the undecidability of BBI.
\end{abstract}

\section{Introduction}

The logic of Bunched Implications (denoted BI) of Pym and O'Hearn [19] is a well-known sub-structural logic which freely combines additive connectives $\wedge, \vee, \rightarrow$ and multiplicative connectives $*, *$. The additives of $\mathrm{BI}$ behave either intuitionistically or classically giving rise to intuitionistic $\mathrm{BI}$ or Boolean $\mathrm{BI}$ (denoted $\mathrm{BBI}$ ). The language of $\mathrm{BI}$, and in particular its composition operators $*$ and $*$, is at the heart of separation and spatial logics frameworks (see [15] for a discussion on these aspects).

It is striking that the proof-theoretical developments on $\mathrm{BI}$ have so far focused mainly on (intuitionistic) BI, especially since the numerous program verification applications of $\mathrm{BI}$ - notably separation logic [9] or spatial logic [3] - are mainly based on its Boolean variant. Intuitionistic $\mathrm{BI}$ has been given a well-behaved proof theory [21] composed of a bunched sequent calculus enjoying cut-elimination since its inception. Later, Galmiche et al. [6] gave Bl a labelled tableaux system from which decidability was derived. On the contrary, the proof theory of $\mathrm{BBI}$ was reduced to the addition of a double negation principle to that of (intuitionistic) Bl, as Pym did in [21]. For long, the main prooftheoretical result was the completeness of the corresponding Hilbert style system [5] and not much more. It was even unknown whether the relational Kripke semantics (corresponding to the Hilbert system) and the partial monoidal
Kripke semantics (corresponding to the labelled tableaux system) define the same notion of validity.

This situation evolved recently with two main families of results. On the one hand, in the spirit of his work with Calcagno on Classical BI [2], Brotherston provided a Display Logic style proof system for relational $\mathrm{BBI}$ and derived a cut-elimination result from this Display framework [1]. He then tried to obtain decidability with syntactic techniques similar to those Restall successfully used in relevant logics [22]. But for some fundamental logical reasons explained in this paper, his attempt was bound to fail. On the other hand, the authors recently obtained a sound and faithful embedding of $\mathrm{BI}$ into $\mathrm{BBI}$ (both defined with their partial deterministic Kripke semantics), illustrating the counter-intuitive fact that Boolean $\mathrm{BI}$ is surprisingly more expressive than intuitionistic $\mathrm{BI}$ [15]. The result is based on the study of the specific properties of the counter-models generated by proof-search in labelled tableaux systems.

Many questions remained open in relation to the proof theory and semantics of BBI. In particular:

1. Do the relational and the partial deterministic Kripke semantics define the same set of valid formulae?

2. Is validity decidable in either of these semantics?

In this paper we solve both questions and give them a negative answer. Indeed, we first show that the notion of invertibility, definable by the BBI formula $\mathcal{I}=\neg(\top * \neg \mathrm{I})$, is not stable by composition in relational Kripke semantics whereas it is in partial deterministic Kripke semantics. Hence the formula $(\mathcal{I} * \mathcal{I}) \rightarrow \mathcal{I}$ distinguishes these two semantics. Then, we prove the main contribution of this paper which is the undecidability of (universal) validity in $\mathrm{BBI}$, be it relational $\mathrm{BBI}$, partial deterministic $\mathrm{BBI}$ or even total deterministic BBI.

We begin by exploring the relation between phase semantics and Kripke semantics in the context of BBI. Compared to the phase semantics of ILL, we characterize the phase semantics of $\mathrm{BBI}$ as trivial because it corresponds to the choice of the least stable closure: the identity closure. 
We point out the direct correspondence between Kripke semantics and trivial phase semantics. From this correspondence, we derive a map from ILL sequents to BBI formulae that is a sound and faithful embedding, as soon as validity in ILL is defined by trivial phase semantics.

On the one hand, it could appear at first that we have only displaced the problem from the language of BBI to the language of ILL. On the other hand, the undecidability of various fragments of ILL is already known, but of course, not with validity defined by trivial phase semantics. It turns out we have changed the question: instead of searching for an undecidable fragment, we have to identify, among the known existing undecidable fragments of ILL, one which is at the same time complete for trivial phase semantics.

This fragment of ILL must include the bang ! connective because IMALL is decidable [13, 17]. As in phase semantics the definition of $\&$ and $\multimap$ does not rely on the closure (as opposed to $\oplus$ and $\otimes$ ), a naive idea would be to keep only those connectives in the desired fragment. If we consider the first fragments of linear logic that were proved undecidable like full propositional linear logic [17] or the $(!, \oplus)$-Horn fragment [11], we observe that, unfortunately, they include both $\otimes$ and $\oplus$. Recently, a fragment of IMELL denoted S-IMELL ${ }_{0}^{\circ}$ has been studied and characterized as equi-decidable to IMELL [4]. This fragment is important to us because it contains neither $\otimes$ nor $\oplus$ and it is very simple. However, decidability for IMELL is still an open question.

It turns out that $\mathrm{S}-\mathrm{IMELL}_{0}^{-}$is indeed complete for trivial phase semantics. We extend its goal-directed proof system [4] with the addition of the \& connective, in the spirit of Kanovich's [11] and Lafont's [12] ideas for encoding Minsky machines in linear logic. The fragment obtained, denoted ILL ${ }_{-0, \& !}^{0}$, is sufficient to encode Minsky machines computations, because the $\&$ connective can be used to simulate forking. We show that, as with s-IMELL ${ }_{0}^{\circ}, I_{L L}^{0}{ }_{-\&, !}$ is still complete for trivial phase semantics.

\section{Non-Deterministic Monoids, ILL and BBI}

In this section, we define $\mathrm{BBI}$ and its non-deterministic (or relational) Kripke semantics, ILL and its non-deterministic phase semantics and establish a semantic link between those two logics: trivial phase semantics.

\subsection{Non-Deterministic Monoids}

Let us consider a set $M$. We denote by $\mathcal{P}(M)$ the powerset of $M$, i.e., its set of subsets. A binary function $\circ: M \times$ $M \longrightarrow \mathcal{P}(M)$ is naturally extended to a binary operator on $\mathcal{P}(M)$ by $X \circ Y=\bigcup\{x \circ y \mid x \in X, y \in Y\}$ for any subsets $X, Y$ of $M$. Using this extension, we can view an element $m$ of $M$ as the singleton set $\{m\}$ and derive the equations $m \circ X=\{m\} \circ X$ and $a \circ b=\{a\} \circ\{b\}$.
Definition 2.1. A non-deterministic (or relational) monoid is a triple $(M, \circ, \epsilon)$ where $\epsilon \in M$ and $\circ: M \times M \longrightarrow \mathcal{P}(M)$ for which the following conditions hold:

1. $\forall a \in M, \epsilon \circ a=\{a\}$ (neutrality)

2. $\forall a, b \in M, a \circ b=b \circ a$ (commutativity)

3. $\forall a, b, c \in M, a \circ(b \circ c)=(a \circ b) \circ c{\text { (associativity })^{1}}^{1}$

The extension of $\circ$ to $\mathcal{P}(M)$ thus induces a commutative monoidal structure with unit element $\{\epsilon\}$ on $\mathcal{P}(M)$.

The term non-deterministic was introduced in [5] in order to emphasize the fact that the composition $a \circ b$ may yield not only one but an arbitrary number of results including the possible incompatibility of $a$ and $b$ in which case $a \circ b=\emptyset$. If $(M, \bullet, \mathrm{e})$ is a (usual) commutative monoid then, defining $a \circ b=\{a \bullet b\}$ and $\epsilon=\mathrm{e}$ induces a non-deterministic monoid $(M, \circ, \epsilon)$. Using the bijection $x \mapsto\{x\}$ mapping elements of $M$ to singletons in $\mathcal{P}(M)$, we can view (usual) commutative monoids as a particular case of non-deterministic monoids (later called total deterministic monoids). Partial monoids can also be represented using the empty set $\emptyset$ as the result of undefined compositions.

The term relational is sometimes used because the map ० : $M \times M \longrightarrow \mathcal{P}(M)$ can equivalently be understood as a ternary relation $-\circ-\ni-: M \times M \times M \longrightarrow\{0,1\}$ through the Curry-Howard isomorphism and the axioms correspond to those of an internal monoid in the category of relations [7]. The two presentations are equivalent but we rather use the monoidal presentation in this paper because of the context of Kripke and phase semantics.

Definition 2.2. Let us consider a non-deterministic monoid $(M, \circ, \epsilon) . \quad$ It is a partial deterministic monoid if for all $x, y \in M$, the composition $x \circ y$ is either empty or a singleton. It is a total deterministic monoid if for all $x, y \in M$, the composition $x \circ y$ is a singleton. The class of nondeterministic (resp. partial deterministic, resp. total deterministic) monoids is denoted ND (resp. PD, resp. TD).

The reader may have noticed that total deterministic monoids (of class TD) exactly correspond to those nondeterministic monoids derived from usual commutative monoids because the composition $\circ$ is a functional relation (exactly one image for each pair of parameters). Obviously, there is also a strict inclusion between those classes of monoids.

\section{Proposition 2.3. $\mathrm{TD} \subsetneq \mathrm{PD} \subsetneq \mathrm{ND}$}

We now exploit the notion of non-deterministic monoid to establish links between the semantics of BBI and ILL.

\subsection{Kripke Semantics for BBI}

The syntax of $\mathrm{BBI}$ is exactly the syntax of $\mathrm{Bl}$ augmented with negation, although negation could be defined

\footnotetext{
${ }^{1}$ Associativity should be understood using the extension of $\circ$ to $\mathcal{P}(M)$.
} 
by $\neg A=A \rightarrow \perp$ like in classical logic. Thus, the formulae of BBI are defined as follows: starting from a set Var, they are freely built using the logical variables in Var, the logical constants in $\{I, \top, \perp\}$, the unary connective $\neg$ or the binary connectives in $\{*, *, \wedge, \vee, \rightarrow\}$. Formally, the set of formulae is described by the following grammar: $A::=v|c| \neg A \mid A$ 米A with $v \in \operatorname{Var}, c \in\{\mathrm{I}, \top, \perp\}$ and 困 $\in\{*, \rightarrow, \wedge, \vee, \rightarrow\}$.

Validity in $\mathrm{BBI}$ has not always been unequivocally defined. Indeed, the initial proposition of Pym [21] was simply to add a double negation principle to the cut-free bunched proof system of BI. But of course, this does not lead to a proof-theoretically well-behaved proof-system for BBI: it does not enjoy cut-elimination, sub-formula property, etc. Then, the syntax of BBI has been used as a foundation for various forms of separation logic with the common property that the additive operator $\rightarrow$ is interpreted classically whereas it is interpreted intuitionistically in BI. The removal of the pre-order in the Kripke semantics is moreover necessary for the interpretation of classical negation $\neg$.

In this paper, we choose to present BBI as a family of logics defined by their Kripke semantics rather than proofsystems. Given a non-deterministic monoid $(M, \circ, \epsilon)$ and an interpretation $\delta: \operatorname{Var} \longrightarrow \mathcal{P}(\mathcal{M})$ of propositional variables, we define the Kripke forcing relation by induction on the structure of formulae:

$$
\begin{gathered}
m \Vdash v \text { iff } m \in \delta(v) \\
m \Vdash \perp \text { iff never } \quad m \Vdash \neg A \text { iff } m \nVdash A \\
m \Vdash \top \text { iff always } \quad m \Vdash I \text { iff } m=\epsilon \\
m \Vdash A \wedge B \text { iff } m \Vdash A \text { and } m \Vdash B \\
m \Vdash A \vee B \text { iff } m \Vdash A \text { or } m \Vdash B \\
m \Vdash A \rightarrow B \text { iff } m \nVdash A \text { or } m \Vdash B \\
m \Vdash A * B \text { iff } \exists a, b, m \in a \circ b \text { and } a \Vdash A \text { and } b \Vdash B \\
m \Vdash A \rightarrow B \text { iff } \forall a, b(b \in m \circ a \text { and } a \Vdash A) \Rightarrow b \Vdash B
\end{gathered}
$$

A formula $F$ is valid in a non-deterministic monoid $(M, \circ, \epsilon)$ if for any interpretation $\delta: \operatorname{Var} \longrightarrow \mathcal{P}(M)$ of propositional variables, the relation $m \Vdash F$ holds for any $m \in M$. A counter-model of the formula $F$ is given by a non-deterministic monoid $(M, \circ, \epsilon)$, an interpretation $\delta: \operatorname{Var} \longrightarrow \mathcal{P}(M)$ and an element $m \in M$ such that $m \nVdash F$.

In some papers, you might find BBI defined by nondeterministic monoidal Kripke semantics [1, 5], in other papers it is defined by partial but deterministic monoidal Kripke semantics and generally separation logic models are particular instances of partial (deterministic) monoids. See [15] for a general discussion about these issues.

Definition 2.4. We denote by $\mathrm{BBI}_{\mathrm{ND}}$ (resp. $\mathrm{BBI}_{\mathrm{PD}}$, resp. $\mathrm{BBI}_{\mathrm{TD}}$ ) the set of formulae of $\mathrm{BBI}$ which are valid in every monoid of the class $\mathrm{ND}$ (resp. $\mathrm{PD}$, resp. TD).

On the proof-theoretic side, we briefly recall that $\mathrm{BBI}_{\mathrm{ND}}$ has been proved sound and complete w.r.t. a Hilbert proofsystem [5] and also, more recently w.r.t. a Display Logic based proof-system [1] enjoying cut-elimination. $\mathrm{BBI}_{\mathrm{PD}}$ can be proved sound and complete w.r.t. the semantic constraints based tableaux proof-system presented in [15] (although only the soundness proof is presented in that particular paper) and the adaptation of this tableaux system to $\mathrm{BBI}_{\mathrm{TD}}$ should be straightforward (contrary to $\mathrm{BBI}_{\mathrm{ND}}$ ).

As it turns out, the three different classes of models ND, $\mathrm{PD}$ and TD define three different logics, i.e., universally valid formulae differ from one class of models to another. The relation of strict inclusion between $\mathrm{BBI}_{\mathrm{ND}}$ and $\mathrm{BBI} \mathrm{PD}_{\mathrm{PD}}$ was, to our knowledge, an undecided proposition.

Theorem 2.5. $\mathrm{BBI}_{\mathrm{ND}} \subsetneq \mathrm{BBI}_{\mathrm{PD}} \subsetneq \mathrm{BBI}_{\mathrm{TD}}$

Proof. The following inclusion relations TD $\subseteq \mathrm{PD} \subseteq \mathrm{ND}$ hold between the classes of models which respectively define those three logics. Hence, only the strictness of the inclusion of validities is not obvious. This strictness is established by upcoming Theorem 2.6 and Proposition 2.7.

Consider the formula $\mathcal{I}=\neg(T * \neg \mathrm{l})$ and a non-deterministic monoid $(M, \circ, \epsilon)$. Since $\mathcal{I}$ does not contain any variable, its Kripke interpretation does not depend on the choice of $\delta$. One can check that for any $x \in M, x \Vdash \mathcal{I}$ iff there exists $x^{\prime} \in M$ s.t. $\epsilon \in x \circ x^{\prime}$. So $\mathcal{I}$ expresses "invertibility" in Kripke semantics. The formula $(\mathcal{I} * \mathcal{I}) \rightarrow \mathcal{I}$ expresses stability of invertibility by monoidal composition.

Theorem 2.6. The formula $(\mathcal{I} * \mathcal{I}) \rightarrow \mathcal{I}$ is valid in every partial deterministic monoid, where $\mathcal{I}=\neg(\top \rightarrow \neg \mathrm{l})$. There exists a non-deterministic monoid which is a counter-model of $(\mathcal{I} * \mathcal{I}) \rightarrow \mathcal{I}$.

Proof. First the counter-model. Consider the non-deterministic monoid $(\{\epsilon, \mathrm{x}, \mathrm{y}\}, \circ, \epsilon)$ uniquely defined by $\mathrm{x} \circ \mathrm{x}=$ $\{\epsilon, \mathrm{y}\}, \mathrm{y} \circ \alpha=\{\mathrm{y}\}$ for any $\alpha \in\{\epsilon, \mathrm{x}, \mathrm{y}\}$ and the conditions $1 \& 2$ of Definition 2.1. Then $\mathrm{x} \Vdash \mathcal{I}$ because there exists $\alpha(\alpha=\mathrm{x})$ such that $\epsilon \in \mathrm{x} \circ \alpha$. On the other hand, $\mathrm{y} \nVdash \mathcal{I}$ because there is no $\alpha$ such that $\epsilon \in \mathrm{y} \circ \alpha$ holds. So, as $\mathrm{y} \in \mathrm{x} \circ \mathrm{x}$, we have $\mathrm{y} \Vdash \mathcal{I} * \mathcal{I}$. Thus y $\nVdash(\mathcal{I} * \mathcal{I}) \rightarrow \mathcal{I}$.

Now let us prove that $(\mathcal{I} * \mathcal{I}) \rightarrow \mathcal{I}$ is valid in every partial deterministic monoid. Let $(M, \circ, \epsilon)$ be a partial deterministic monoid. Let us choose $a \in M$ and prove that $a \Vdash(\mathcal{I} * \mathcal{I}) \rightarrow \mathcal{I}$. So we suppose $a \Vdash \mathcal{I} * \mathcal{I}$ holds and we have to prove $a \Vdash \mathcal{I}$. As $a \Vdash \mathcal{I} * \mathcal{I}$, there exist $b, c \in M$ such that $a \in b \circ c, b \Vdash \mathcal{I}$ and $c \Vdash \mathcal{I}$. Thus there exist $b^{\prime}, c^{\prime} \in M$ such that $\epsilon \in b \circ b^{\prime}$ and $\epsilon \in c \circ c^{\prime}$. As $\circ$ is (partial) deterministic, we have $b \circ b^{\prime}=\{\epsilon\}, c \circ c^{\prime}=\{\epsilon\}$ and $b \circ c=\{a\}$. Thus we have $\left(b \circ b^{\prime}\right) \circ\left(c \circ c^{\prime}\right)=\{\epsilon\} \circ\{\epsilon\}=\{\epsilon\}$.

If $b^{\prime} \circ c^{\prime}=\emptyset$ then we would have $(b \circ c) \circ\left(b^{\prime} \circ c^{\prime}\right)=$ $\{a\} \circ \emptyset=\emptyset$ but also $\left(b \circ b^{\prime}\right) \circ\left(c \circ c^{\prime}\right)=\{\epsilon\}$ and thus $\emptyset=$ $\{\epsilon\}$ by associativity/commutativity, which is absurd. Thus $b^{\prime} \circ c^{\prime}=\left\{a^{\prime}\right\}$ and we obtain $(b \circ c) \circ\left(b^{\prime} \circ c^{\prime}\right)=\{a\} \circ\left\{a^{\prime}\right\}=$ $a \circ a^{\prime}$ and then $a \circ a^{\prime}=\{\epsilon\}$ by associativity/commutativity. Hence, $\epsilon \in a \circ a^{\prime}$ and $a \Vdash \mathcal{I}$. 
The formula $(\neg \mid \rightarrow \perp) \rightarrow I$ is inspired from the example given to establish the incompleteness of (total) monoidal Kripke semantics w.r.t. (intuitionistic) BI (see [21] page 63).

Proposition 2.7. The formula $(\neg \mathrm{I} * \perp) \rightarrow \mathrm{I}$ is valid in every total deterministic monoid. There exists a partial deterministic monoid which is a counter-model to $(\neg \mathrm{I} * \perp) \rightarrow \mathrm{I}$.

The proof can be found in [16] (Appendix A). Having defined the Kripke semantics of BBI within the framework of non-deterministic monoids, let us consider nondeterministic phase semantics for ILL.

\subsection{Non-Deterministic Phase Spaces for ILL}

The formulae of ILL are defined by the following grammar: $A::=v|c| ! A \mid A$ 困 $A$ with $v \in \operatorname{Var}, c \in\{1, \top, \perp\}^{2}$ and $\Theta \in\{\otimes, \multimap, \&, \oplus\}$. A sequent is a pair denoted $\Gamma \vdash A$ where $\Gamma$ is a multiset of formulae and $A$ is a single formula. The sequent calculus Sill (see Figure 1) is provided for ILL and the set of derivable sequents is the least set closed under its rules. Notice that $\Gamma, \Delta$ denote multisets of formulae and $A, B, C$ denote formulae. In rule $\left\langle!_{R}\right\rangle, ! \Gamma$ denotes the multiset $! \Gamma=! A_{1}, \ldots, ! A_{k}$ if $\Gamma=A_{1}, \ldots, A_{k}$.

The notion of sequent calculus proof is defined as usual: an ordered tree where each node together with its sons correspond to an instance of one of the rules of Sill. Hence, a sequent is derivable if and only if there exists a proof of it in Sill. By historical definition of ILL [8], the sequents which are provable in Sill are exactly the valid sequents of ILL, and a formula $A$ of ILL is valid if $\vdash A$ is a valid sequent.

We extend the notion of intuitionistic phase space [8] to non-deterministic monoids and show that this semantic interpretation is sound and complete w.r.t. Sill, and thus equivalent to the original notion (see Corollary 2.12).

Definition 2.8. $A$ non-deterministic (intuitionistic) phase space is given by a non-deterministic monoid $(M, \circ, \epsilon)=$ $\mathcal{M}$ together with a stable closure operator $(\cdot)^{\diamond}: \mathcal{P}(M) \longrightarrow$ $\mathcal{P}(M)$ and a sub-monoid $K$ included in $J=\{x \in M \mid x \in$ $\left.\{\epsilon\}^{\diamond} \cap(x \circ x)^{\diamond}\right\}$.

The closure property corresponds to the condition $X \subseteq$ $Y^{\diamond}$ iff $X^{\diamond} \subseteq Y^{\diamond}$ for any $X, Y \in \mathcal{P}(M)$. We recall that the monoidal composition $\circ$ is naturally extended to $\mathcal{P}(M)$ by $X \circ Y=\bigcup\{x \circ y \mid x \in X, y \in Y\}$ providing a (commutative) monoidal structure on $\mathcal{P}(M)$ with unit $\{\epsilon\}$.

The stability property ${ }^{3}$ corresponds to the condition $X^{\diamond} \circ$ $Y^{\diamond} \subseteq(X \circ Y)^{\diamond}$ for any $X, Y \in \mathcal{P}(M)$. A subset $X$ of $M$ is $(\cdot)^{\diamond}$-closed (or simply closed when the closure is obvious from the context) if $X^{\diamond}=X$ or equivalently $X^{\diamond} \subseteq X$. The set of closed subsets is denoted $\mathcal{M}^{\diamond}=\{X \in \mathcal{P}(M) \mid$

\footnotetext{
${ }^{2}$ Sometimes the neutral of $\oplus$ is denoted 0 , but we favor $\perp$ as in [23].

${ }^{3}$ A stable closure is a quantic nucleus in quantale theory [24]. The "stability" property itself seems to have no well-established terminology.
}

$\left.X^{\diamond}=X\right\}$, not to be confused with $M^{\diamond}$ where $M$ is viewed as the (total) subset of $M$ (and in this case, $M^{\diamond}=M$ ). Any intersection of closed subsets is a closed subset and thus $\mathcal{M}^{\diamond}$ is invariant under arbitrary intersections, inducing a complete lattice structure on $\left(\mathcal{M}^{\diamond}, \subseteq\right)$.

The set $K$ is just a sub-monoid of $\mathcal{M}$ included in $J$, i.e., $K$ verifies $\epsilon \in K \subseteq J$ and $K \circ K \subseteq K$. Let $\multimap$ be the binary operator on $\mathcal{P}(M)$ defined by $X \multimap Y=\{k \in$ $M \mid k \circ X \subseteq Y\}$. In the lattice $(\mathcal{P}(M), \subseteq)$, the operator $\multimap$ is contra-variant in its first parameter and co-variant in its second and $K \subseteq X \multimap Y$ iff $K \circ X \subseteq Y$ for any $K, X, Y \in \mathcal{P}(M)$. By stability of the closure operator $(\cdot)^{\diamond}$, the subset $X \multimap Y$ is closed as soon as $Y$ is closed and $X \multimap Y^{\diamond}=X^{\diamond} \multimap Y^{\diamond}$ for any $X, Y \in \mathcal{P}(M)$.

We see that we have a (quite direct) generalization of the usual notion of phase space in the case where the monoid is neither supposed to be total nor deterministic. In the particular case of total deterministic monoids, we recover the usual notion of phase space.

The interpretation of ILL connectives is done in the following way. Given an interpretation $\llbracket \cdot \rrbracket:$ Var $\longrightarrow \mathcal{M}^{\diamond}$ of logical variables into closed subsets, this interpretation is extended to all the formulae of ILL by structural induction as follows:

$$
\begin{aligned}
& \llbracket \perp \rrbracket=\emptyset^{\diamond} \\
& \llbracket \top \rrbracket=M \\
& {\left[[1]=\{\epsilon\}^{\diamond}\right.} \\
& \llbracket ! A \rrbracket=(K \cap \llbracket A \rrbracket)^{\diamond} \\
& \begin{array}{l}
\llbracket A \oplus B \rrbracket=(\llbracket A \rrbracket \cup \llbracket B \rrbracket)^{\diamond} \\
\llbracket A \& B \rrbracket=\llbracket A \rrbracket \cap \llbracket B \rrbracket \\
\llbracket A \otimes B \rrbracket=(\llbracket A \rrbracket \circ \llbracket B \rrbracket)^{\diamond} \\
\llbracket A \multimap B \rrbracket=\llbracket A \rrbracket \multimap \llbracket B \rrbracket
\end{array}
\end{aligned}
$$

Again, when the interpretation is done in a total deterministic monoid, we obtain exactly the same value for $\llbracket A \rrbracket$ as in the usual phase semantics interpretation. A sequent $A_{1}, \ldots, A_{k} \vdash B$ of ILL is valid in the interpretation $\left.\llbracket \cdot\right]$ if $\llbracket A_{1} \rrbracket \circ \cdots \circ \llbracket A_{k} \rrbracket \subseteq \llbracket B \rrbracket$. We recall the soundness theorem which states that provability in Sill entails semantic validity in non-deterministic intuitionistic phase semantics.

Theorem 2.9. If the sequent $A_{1}, \ldots, A_{k} \vdash B$ has a proof in Sill, then the inclusion $\llbracket A_{1} \rrbracket \circ \cdots \circ \llbracket A_{k} \rrbracket \subseteq \llbracket B \rrbracket$ holds.

Proof. The proof of this theorem can be done directly by generalizing the soundness proof of usual phase semantics [8], or else, as done in [16] (appendix B), by using the algebraic semantic characterization of ILL of [23].

Definition 2.10. We denote by $\mathrm{ILL}_{p}$ the set of sequents which have a proof in Sill. We denote by ILL $\mathrm{LD}_{\mathrm{ND}}$ (resp. $\mathrm{ILL}_{\mathrm{PD}}$, resp. ILL $\mathrm{LL}_{\mathrm{TD}}$ ) the set of sequents which are valid in every non-deterministic phase semantic interpretation where the base monoid is of the class $\mathrm{ND}$ (resp. PD, resp. TD).

Let us consider the following inclusion sequence:

$$
\mathrm{ILL}_{p} \subseteq \mathrm{ILL}_{\mathrm{ND}} \subseteq \mathrm{ILL}_{\mathrm{PD}} \subseteq \mathrm{ILL}_{\mathrm{TD}} \subseteq \mathrm{ILL}_{p}
$$




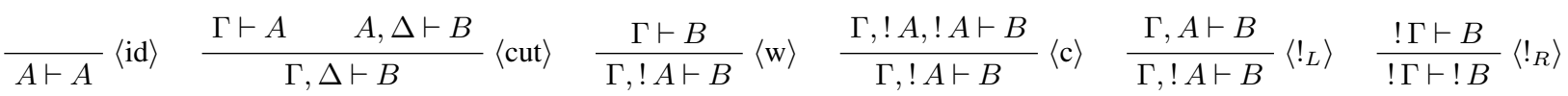

$$
\begin{aligned}
& \frac{\Gamma, A, B \vdash C}{\Gamma, A \otimes B \vdash C}\left\langle\otimes_{L}\right\rangle \quad \frac{\Gamma \vdash A \quad \Delta, B \vdash C}{\Gamma, \Delta, A \multimap B \vdash C}\left\langle\multimap_{L}\right\rangle \quad \frac{\Gamma \vdash A \quad \Delta \vdash B}{\Gamma, \Delta \vdash A \otimes B}\left\langle\otimes_{R}\right\rangle \quad \frac{\Gamma, A \vdash B}{\Gamma \vdash A \multimap B}\left\langle\multimap_{R}\right\rangle
\end{aligned}
$$

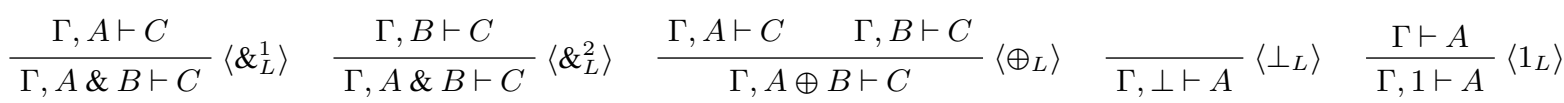

$$
\begin{aligned}
& \frac{\Gamma \vdash A \quad \Gamma \vdash B}{\Gamma \vdash A \& B}\left\langle \&_{R}\right\rangle \quad \frac{\Gamma \vdash A}{\Gamma \vdash A \oplus B}\left\langle\oplus_{R}^{1}\right\rangle \quad \frac{\Gamma \vdash B}{\Gamma \vdash A \oplus B}\left\langle\oplus_{R}^{2}\right\rangle \quad \frac{}{\Gamma \vdash \top}\left\langle\top_{R}\right\rangle \quad \frac{}{\vdash 1}\left\langle 1_{R}\right\rangle
\end{aligned}
$$

Figure 1. Sequent calculus Sill for ILL

The first inclusion $\mathrm{ILL}_{p} \subseteq \mathrm{ILL}_{\mathrm{ND}}$ is given by Theorem 2.9. The two following inclusions ILL $\mathrm{IL}_{\mathrm{ND}} \subseteq \mathrm{ILL}$ PD $\subseteq \mathrm{ILL}_{\mathrm{TD}}$ are obvious consequences of the inclusions $\mathrm{TD} \subseteq \mathrm{PD} \subseteq \mathrm{ND}$ between classes of non-deterministic monoids. The last inclusion $\mathrm{ILL}_{\mathrm{TD}} \subseteq \mathrm{ILL}_{p}$ is just a reformulation of the completeness of the (usual) phase semantics w.r.t. Sill.

Proposition 2.11. If $\Gamma \vdash A$ is valid in every non-deterministic phase semantic interpretation $\left(M, \circ, \epsilon,(\cdot)^{\diamond}, K, \llbracket \cdot \rrbracket\right)$ with $(M, \circ, \epsilon)$ of the class TD, then $\Gamma \vdash A$ has a proof in Sill.

Proof. Total deterministic monoids (of the class TD) are in one to one correspondence with (usual) commutative monoids and this correspondence trivially extends to phase semantics. The result is simply a reformulation of the completeness of usual phase semantics $[8,23]$ w.r.t. ILL.

Corollary 2.12. $\mathrm{ILL}_{p}=I L L_{\mathrm{ND}}=I \mathrm{LL}_{\mathrm{PD}}=\mathrm{IL} \mathrm{L}_{\mathrm{TD}}$ and non-deterministic intuitionistic phase semantics is both sound and complete w.r.t. Sill.

Proof. With Proposition 2.11, we have closed the circular inclusion sequence (1). In particular ILL $\mathrm{IL}_{p}=\mathrm{ILL}_{\mathrm{ND}}$.

\subsection{Trivial Phase vs. Kripke Semantics}

In this section, we define trivial phase semantics which is a particular case of phase semantics that can be viewed as Kripke semantics put in a particular form.

Definition 2.13. Given a non-deterministic monoid $\mathcal{M}=$ $(M, \circ, \epsilon)$, the trivial phase space is defined by taking the identity map on $\mathcal{P}(M)$ as closure operator (i.e., for all $X \in$ $\left.\mathcal{P}(M), X^{\diamond}=X\right)$ and by taking $K=\{\epsilon\}$.

It is clear that the identity on $\mathcal{P}(M)$ is both a closure and stable. Obviously also, $K=\{\epsilon\}$ verifies the conditions $\epsilon \in K \subseteq J$ and $K \circ K \subseteq K{ }^{4}$ In a trivial phase space,

\footnotetext{
${ }^{4}$ In fact, there is no other choice for $K$ because $J=\{x \in M \mid x \in$ $\left.\{\epsilon\}^{\diamond} \cap(x \circ x)^{\diamond}\right\}=\{\epsilon\}$ when $(\cdot)^{\diamond}$ is the identity map on $\mathcal{P}(M)$.
}

every subset of $M$ is closed and thus $\mathcal{M}^{\diamond}=\mathcal{P}(M)$. The interpretation of ILL connectives becomes:

$$
\begin{aligned}
& \llbracket \perp \rrbracket=\emptyset \\
& \llbracket \top \rrbracket=M \\
& \llbracket 1 \rrbracket=\{\epsilon\} \\
& \llbracket ! A \rrbracket=\{\epsilon\} \cap \llbracket A \rrbracket \\
& \llbracket A \oplus B \rrbracket=\llbracket A \rrbracket \cup \llbracket B \rrbracket \\
& \llbracket A \& B \rrbracket=\llbracket A \rrbracket \cap \llbracket B \rrbracket \\
& \llbracket A \otimes B \rrbracket=\llbracket A \rrbracket \circ \llbracket B \rrbracket \\
& \llbracket A \multimap B \rrbracket=\llbracket A \rrbracket \multimap \llbracket B \rrbracket
\end{aligned}
$$

Replacing $1 / \mathrm{I}, \oplus / \vee, \& / \wedge, \otimes / *$ and $\multimap / *$ in the previous equations and defining $\llbracket A \rightarrow B \rrbracket=M \backslash \llbracket A \rrbracket \cup \llbracket B \rrbracket$ and $\llbracket \neg A \rrbracket=M \backslash \llbracket A \rrbracket$ provides a complete (non-deterministic) trivial phase semantics to $\mathrm{BBI}$, in direct correspondence to its Kripke semantics. Thus, there is an embedding of the connectives of ILL into BBI, which can be formalized with the following inductively defined map $(\cdot)^{\circledast}: \mathrm{ILL} \longrightarrow \mathrm{BBI}$ :

$$
\begin{aligned}
& v^{\circledast}=v \quad \text { for } v \in \operatorname{Var} \\
& \perp^{\circledast}=\perp \quad(A \oplus B)^{\circledast}=A^{\circledast} \vee B^{\circledast} \\
& \bar{\top}^{\circledast}=\top \quad(A \& B)^{\circledast}=A^{\circledast} \wedge B^{\circledast} \\
& 1^{\circledast}=\mathrm{I} \quad(A \otimes B)^{\circledast}=A^{\circledast} * B^{\circledast} \\
& (! A)^{\circledast}=\mathrm{I} \wedge A^{\circledast} \quad(A \multimap B)^{\circledast}=A^{\circledast} * B^{\circledast}
\end{aligned}
$$

Lemma 2.14. In trivial phase semantics, if the phase interpretation $\llbracket \cdot \rrbracket: \operatorname{Var} \longrightarrow \mathcal{M}^{\diamond}$ and the Kripke interpretation $\delta: \operatorname{Var} \longrightarrow \mathcal{P}(M)$ are identical maps then the phase semantics and the Kripke semantics are in relation as follows:

$$
\forall F \in \mathrm{ILL}, \forall m \in M, m \in \llbracket F \rrbracket \text { iff } m \Vdash F^{\circledast}
$$

Proof. By structural induction on $F$. We only consider the case $F=A \otimes B$. Let $m \in M$, we have $m \in \llbracket A \otimes B \rrbracket$ iff $m \in \llbracket A \rrbracket \circ \llbracket B \rrbracket$ iff $\exists a \in \llbracket A \rrbracket, \exists b \in \llbracket B \rrbracket, m \in a \circ b$ iff $\exists a, b,\left(a \Vdash A^{\circledast}\right.$ and $b \Vdash B^{\circledast}$ and $\left.m \in a \circ b\right)$ iff $m \Vdash$ $A^{\circledast} * B^{\circledast}$ iff $m \Vdash(A \otimes B)^{\circledast}$.

So if the interpretation of logical variables coincide, trivial phase semantics and Kripke semantics correspond to each other through the map $(\cdot)^{\circledast}$. Given a sequence $\mathcal{S}=A_{1}, \ldots, A_{k}$ of formulae of ILL, we define $\mathcal{S}^{\circledast}$ by structural induction on $\mathcal{S}$ :

$$
()^{\circledast}=\mathrm{I} \quad\left(A_{1}, \ldots, A_{k+1}\right)^{\circledast}=A_{1}^{\circledast} *\left(A_{2}, \ldots, A_{k+1}\right)^{\circledast}
$$


When $\llbracket \cdot \rrbracket$ and $\delta$ are identical maps on propositional variables, it is then straightforward to prove this equivalence by induction on $k$ :

$$
m \in \llbracket A_{1} \rrbracket \circ \cdots \circ \llbracket A_{k} \rrbracket \quad \text { iff } m \Vdash\left(A_{1}, \ldots, A_{k}\right)^{\circledast}
$$

Lemma 2.15. If $A_{1}, \ldots, A_{k} \vdash B$ has a proof in Sill then the formula $\left(A_{1}, \ldots, A_{k}\right)^{\circledast} \rightarrow B^{\circledast}$ belongs to $\mathrm{BBI} \mathrm{ND}_{\mathrm{ND}}$.

Proof. Let us suppose that the sequent $A_{1}, \ldots, A_{k} \vdash B$ is provable in Sill. We show that $\left(A_{1}, \ldots, A_{k}\right)^{\circledast} \rightarrow B^{\circledast}$ belongs to $\mathrm{BBI}_{\mathrm{ND}}$. Let us consider a non-deterministic monoid $(M, \circ, \epsilon)$ and an interpretation $\delta: \operatorname{Var} \longrightarrow \mathcal{P}(M)$. For the non-deterministic trivial phase space associated to $(M, \circ, \epsilon)$, we choose the phase interpretation $\llbracket v \rrbracket=\delta(v)$ for any variable $v \in \operatorname{Var}$. By soundness of non-deterministic phase semantics (see Theorem 2.9), we obtain the inclusion $\llbracket A_{1} \rrbracket \circ \cdots \circ \llbracket A_{k} \rrbracket \subseteq \llbracket B \rrbracket$. Then, by (2) and (3), for any $m \in M$ we have $m \Vdash\left(A_{1}, \ldots, A_{k}\right)^{\circledast} \rightarrow B^{\circledast}$. As this holds for any non-deterministic Kripke interpretation, we deduce that $\left(A_{1}, \ldots, A_{k}\right)^{\circledast} \rightarrow B^{\circledast}$ belongs to $\mathrm{BBI}_{\mathrm{ND}}$.

Lemma 2.16. If the sequent $A_{1}, \ldots, A_{k} \vdash B$ has a countermodel in total deterministic trivial phase semantics then the formula $\left(A_{1}, \ldots, A_{k}\right)^{\circledast} \rightarrow B^{\circledast}$ does not belong to $\mathrm{BBI}_{\mathrm{TD}}$.

Proof. Let us suppose that there exists a total deterministic monoid $(M, \circ, \epsilon)$ (in TD) and a trivial phase semantics interpretation $\llbracket \cdot \rrbracket: \operatorname{Var} \longrightarrow \mathcal{P}(M)$ such that $\llbracket A_{1} \rrbracket \circ \cdots \circ$ $\llbracket A_{k} \rrbracket \nsubseteq \llbracket B \rrbracket$. Considering the Kripke interpretation defined by $\delta(v)=\llbracket v \rrbracket$ for any $v \in$ Var, by equivalences (2) and (3), there exists $m \in M$ such that $m \nVdash\left(A_{1}, \ldots, A_{k}\right)^{\circledast} \rightarrow B^{\circledast}$. So $\left(A_{1}, \ldots, A_{k}\right)^{\circledast} \rightarrow B^{\circledast}$ has a Kripke counter-model in $\mathrm{TD}$. Hence, this formula does not belong to $\mathrm{BBI} \mathrm{TD}_{\mathrm{TD}}$.

\section{The Undecidability of Boolean BI}

From the preceding results, we establish the undecidability of BBI. We define a reverse map from multisets of formulae of ILL into lists of formulae by choosing an arbitrary computable total order among the formulae of ILL (e.g. lexicographic ordering). For any multiset $\Gamma$ of formulae of ILL, there exists a unique and computable ordered sequence of formulae $A_{1}, \ldots, A_{k}$ s.t. $\Gamma=\left\{A_{1}, \ldots, A_{k}\right\}$ and we define $\Gamma^{\circledast}=\left(A_{1}, \ldots, A_{k}\right)^{\circledast}$. The map $(\cdot)^{\circledast}:$ ILL $\longrightarrow$ BBI defined by $(\Gamma \vdash B) \mapsto\left(\Gamma^{\circledast} \rightarrow B^{\circledast}\right)$ is thus a computable map from sequents of ILL into formulae of BBI.

We introduce the key result that links undecidability in ILL and in BBI. The fragment ILL ${ }_{-\infty, \&, !}^{0}$ is a recursive subset of the set of sequents of ILL (see Definition 3.4).

Theorem 3.1. In ILL ${ }_{-0, \&, !}^{0}$ validity is both undecidable and complete for total deterministic trivial phase semantics.

The proof of this theorem spans over the remaining sections of the paper (see Theorems 3.7 and 3.14).
Theorem 3.2. Let $x \in\{\mathrm{ND}, \mathrm{PD}, \mathrm{TD}\}$. The restricted map $(\cdot)^{\circledast}: \mathrm{ILL}_{-\circ, \&, !}^{0} \longrightarrow \mathrm{BBI}_{x}$ is a sound and faithful embedding.

Proof. Let us consider a given sequent $\Gamma \vdash B$ of ILL ${ }_{-\infty, \&, !}^{0}$. Let $\Gamma=\left\{A_{1}, \ldots, A_{k}\right\}$ where the sequence $A_{1}, \ldots, A_{k}$ is sorted according to the previously chosen total order. Then $(\Gamma \vdash B)=\left(A_{1}, \ldots, A_{k} \vdash B\right)$ and $(\Gamma \vdash B)^{\circledast}=$ $\left(A_{1}, \ldots, A_{k}\right)^{\circledast} \rightarrow B^{\circledast}$. On the one hand, if $\Gamma \vdash B$ is valid in ILL, then it has a proof in Sill and thus, according to Lemma 2.15, the formula $\Gamma^{\circledast} \rightarrow B^{\circledast}$ belongs to $\mathrm{BBI}_{\mathrm{ND}}$. It thus belongs to $\mathrm{BBI}_{x}$ because $\mathrm{BBI}_{\mathrm{ND}} \subseteq \mathrm{BBI}_{x}$ holds (Theorem 2.5). On the other hand, if $\Gamma \vdash B$ is invalid in ILL, then as it belongs to the fragment ILL ${ }_{-\circ, \& !}^{0}$ which is complete w.r.t. total deterministic trivial phase semantics, it has a counter-model in this semantics. Hence by Lemma 2.16, the formula $\Gamma^{\circledast} \rightarrow B^{\circledast}$ does not belong to $\mathrm{BBI}_{\mathrm{TD}}$. Thus, it does not belong to $\mathrm{BBI}_{x}$ either because $\mathrm{BBI}_{x} \subseteq \mathrm{BBI}_{\mathrm{TD}}$ holds (Theorem 2.5).

Theorem 3.3. (Universal) validity in the logic $\mathrm{BBI}_{\mathrm{ND}}$ (resp. $\mathrm{BBI}_{\mathrm{PD}}$, resp. $\mathrm{BBI}_{\mathrm{TD}}$ ) is undecidable.

Proof. For any $x \in\{\mathrm{ND}, \mathrm{PD}, \mathrm{TD}\}$, by Theorem 3.2, a decision procedure for $\mathrm{BBI}_{x}$ would lead to a decision procedure for ILL ${ }_{-o, \&, !}^{0}$ by composition with the obviously computable map $(\cdot)^{\circledast}$, which contradicts Theorem 3.1.

Before we describe the fragment ILL ${ }_{-0, \& !}$ and the proof of Theorem 3.1, we wish to point out the inclusion sequence $\mathrm{ILL}_{\mathrm{ND}}^{t} \subseteq \mathrm{ILL}_{\mathrm{PD}}^{t} \subseteq \mathrm{ILL}_{\mathrm{TD}}^{t}$ as a remaining open question where ILL $L_{x}^{t}$ is defined by trivial phase semantics with the monoid belonging to the class $x \in\{\mathrm{ND}, \mathrm{PD}, \mathrm{TD}\}$. The question is: are these two inclusions strict? Indeed, the counter-examples of Theorem 2.6 and Proposition 2.7 cannot be used because both formulae contain a negation.

\subsection{A Trivially Complete Fragment of ILL}

We define and characterize an extension of the fragment s-IMELL ${ }_{0}^{-}$of ILL [4] which we denote ILL ${ }_{-0, \& ! !}^{0}$. We provide a simple goal-directed proof system, denoted Gill ${ }^{0}$, which is itself an extension of the goal-directed proof system of s-IMELL -0 , obtained by the addition of a new additive rule. Then we show that the proof system Gill ${ }^{0}$ and trivial phase semantics are both sound and complete w.r.t. the fragment ILL ${ }_{-\infty, \& ! !}^{0}$.

Definition 3.4. A formula of ILL is $(\multimap, \&)$-elementary if it is of the form $u \multimap v,(u \multimap v) \multimap w, u \multimap(v \multimap w)$ or $(u \& v) \multimap$ $w$ where $u, v$ and $w$ are logical variables. The sequents of the fragment ILL ${ }_{-\infty, \&, !}^{0}$ are those of the form $! \Sigma, \Gamma \vdash c$ where $\Gamma$ is a multiset of variables, $c$ is a variable and $\Sigma$ is a multiset of $(-, \&)$-elementary formulae.

From this definition, it is obvious that membership in the fragment $\mathrm{ILL}_{-\infty, \&, !}^{0}$ is a recursive property. Compared 


$$
\begin{aligned}
& \frac{! \Sigma, \Gamma \vdash a}{! \Sigma, a \vdash a}\langle\operatorname{Ax}\rangle \quad \frac{! \Sigma, \Gamma, a \vdash b}{! \Sigma, \Gamma \vdash b} a \multimap b \in \Sigma \quad \frac{!}{! \Sigma, \Gamma \vdash c}(a \multimap b) \multimap c \in \Sigma \\
& \frac{! \Sigma, \Gamma \vdash a \quad ! \Sigma, \Delta \vdash b}{! \Sigma, \Gamma, \Delta \vdash c} a \multimap(b \multimap c) \in \Sigma \quad \frac{! \Sigma, \Gamma \vdash a \quad ! \Sigma, \Gamma \vdash b}{! \Sigma, \Gamma \vdash c}(a \& b) \multimap c \in \Sigma
\end{aligned}
$$

Figure 2. Gill ${ }^{0}$ : a goal-directed sequent calculus for $\operatorname{ILL}_{-\infty, \&, !}^{0}$

to $\mathrm{s}-\mathrm{IMELL}_{0}^{-}$, the only new form is $(u \& v) \multimap w$. The validity of sequents in ILL ${ }_{-\infty, \&, !}^{0}$ can be established using the proof system Sill but we rather provide an alternative goal-directed proof system called Gill ${ }^{0}$ in Figure 2. Apart for the axiom rule $\langle A x\rangle$, each other rule $\langle\multimap\rangle,\langle(\multimap) \multimap\rangle$, $\langle\multimap(-0)\rangle$ or $\langle(\&) \multimap\rangle$ is named according to the form of its side condition. Compared to s-IMELL ${ }_{0}^{-}$, the only new rule is $\langle(\&)-\rangle$. In [4], the authors did not provide a proof of soundness/completeness of the system s-IMELL ${ }_{0}^{-}$, leaving it to the reader. Here we present a full proof of soundness/completeness for our extension Gill ${ }^{0}$ not only to please the reader but also to derive completeness of the fragment w.r.t. trivial phase semantics.

Hence, even though validity in $\mathrm{ILL}_{-\infty, \&, !}^{0}$ is the same as in the whole ILL (established for instance by a proof in Sill), here we show that in this specific fragment, validity is also sound and complete both w.r.t. the system Gill ${ }^{0}$ and w.r.t. total deterministic trivial phase semantics.

Lemma 3.5. Every proof of a sequent in Gill ${ }^{0}$ can be transformed into a proof (of the same sequent) which uses only rules $\langle\mathrm{id}\rangle,\langle\mathrm{w}\rangle,\langle\mathrm{c}\rangle,\left\langle\multimap_{L}\right\rangle,\left\langle\multimap_{R}\right\rangle,\left\langle!_{L}\right\rangle$ and $\left\langle \&_{R}\right\rangle$ of Sill.

Lemma 3.6. If the sequent $! \Sigma, \Gamma \vdash$ c of $\mathrm{ILL}_{-0, \&, !}^{0}$ is valid in every total deterministic trivial phase semantic interpretation then it has a proof in Gill ${ }^{0}$.

Proof. The proof of Lemma 3.5 can be found in [16] (Appendix C). For the proof of Lemma 3.6, we apply a technique similar to the one of Okada [20] for obtaining strong completeness through phase semantics. Let us consider a fixed multiset $\Sigma$ of $(\multimap, \&)$-elementary formulae. We consider the free commutative monoid over the set of logical variables, i.e., the set of finite multisets of variables endowed with multiset addition (denoted by the comma) as monoidal composition and with the empty multiset (denoted $\epsilon=\lfloor\emptyset\rfloor$ ) as neutral element. We write $\lfloor a, a, b\rfloor$ for the multiset composed of two occurrences of $a$ and one of $b$. Let us define the total deterministic monoid $(M, \circ, \epsilon=\lfloor\emptyset\rfloor)$ where $M$ is the set of finite multisets of variables and $\circ: M \times M \longrightarrow \mathcal{P}(M)$ is defined by $\lfloor\Gamma\rfloor \circ\lfloor\Delta\rfloor=\{\lfloor\Gamma, \Delta\rfloor\}$.

We define the following semantic interpretation in the trivial phase space based on $(M, \circ, \epsilon)$ :

$$
\llbracket c \rrbracket=\left\{\lfloor\Gamma\rfloor \mid ! \Sigma, \Gamma \vdash c \text { has a proof in Gill }{ }^{0}\right\} \text { for } c \in \text { Var }
$$

Let us now show that $\epsilon \in \llbracket \sigma \rrbracket$ holds for any $\sigma \in \Sigma$. We pick one $\sigma \in \Sigma$ and proceed by case analysis.

If $\sigma=u \multimap v$. Then $\epsilon \in \llbracket u \multimap v \rrbracket$ iff $\epsilon \circ \llbracket u \rrbracket \subseteq \llbracket v \rrbracket$ iff $\llbracket u \rrbracket \subseteq \llbracket v \rrbracket$. So let us consider one $\lfloor\Gamma\rfloor$ such that $\lfloor\Gamma\rfloor \in$ $\llbracket u \rrbracket$. Let us prove that $\lfloor\Gamma\rfloor \in \llbracket v \rrbracket$. By definition of $\llbracket u \rrbracket$, the sequent $! \Sigma, \Gamma \vdash u$ has a proof in Gill ${ }^{0}$. Then, by rule $\langle\multimap\rangle$, the sequent $! \Sigma, \Gamma \vdash v$ has a proof in Gill ${ }^{0}$. So we deduce $\lfloor\Gamma\rfloor \in \llbracket v \rrbracket$. Hence $\llbracket u \rrbracket \subseteq \llbracket v \rrbracket$ and we obtain $\epsilon \in \llbracket \sigma \rrbracket$.

If $\sigma=(u \multimap v) \multimap w$. We have $\epsilon \in \llbracket(u \multimap v) \multimap w \rrbracket$ iff $\llbracket u \rrbracket \multimap \llbracket v \rrbracket \subseteq \llbracket w \rrbracket$. Let use choose $\lfloor\Gamma\rfloor \in \llbracket u \rrbracket \multimap \llbracket v \rrbracket$. Then $\{\lfloor\Gamma\rfloor\} \circ \llbracket u \rrbracket \subseteq \llbracket v \rrbracket$. By rule $\langle\mathrm{Ax}\rangle, ! \Sigma, u \vdash u$ has a proof in Gillo and thus $\lfloor u\rfloor \in \llbracket u \rrbracket$. Thus $\{\lfloor\Gamma, u\rfloor\}=\lfloor\Gamma\rfloor \circ\lfloor u\rfloor \subseteq \llbracket v \rrbracket$. Then $! \Sigma, \Gamma, u \vdash v$ has a proof in Gill ${ }^{\text {. }}$. By rule $\langle(\multimap)-\rangle$, $! \Sigma, \Gamma \vdash w$ has a proof in Gill ${ }^{0}$. We conclude $\lfloor\Gamma\rfloor \in \llbracket w \rrbracket$. Thus $\llbracket u \rrbracket \multimap \llbracket v \rrbracket \subseteq \llbracket w \rrbracket$ holds, hence $\epsilon \in \llbracket \sigma \rrbracket$.

If $\sigma=u \multimap(v \multimap w)$. We have $\epsilon \in \llbracket u \multimap(v \multimap w) \rrbracket$ iff $\llbracket u \rrbracket \circ \llbracket v \rrbracket \subseteq \llbracket w \rrbracket$. Let us choose $\lfloor\Gamma\rfloor \in \llbracket u \rrbracket$ and $\lfloor\Delta\rfloor \in$ $\llbracket v \rrbracket$ and let us prove $\lfloor\Gamma\rfloor \circ\lfloor\Delta\rfloor \subseteq \llbracket w \rrbracket$. Both $! \Sigma, \Gamma \vdash u$ and $! \Sigma, \Delta \vdash v$ have a proof in Gill ${ }^{0}$. By rule $\langle\multimap(\multimap)\rangle$, the sequent $! \Sigma, \Gamma, \Delta \vdash w$ has a proof in Gill ${ }^{0}$. Thus $\lfloor\Gamma\rfloor \circ\lfloor\Delta\rfloor=$ $\{\lfloor\Gamma, \Delta\rfloor\} \subseteq \llbracket w \rrbracket$. We deduce $\llbracket u \rrbracket \circ \llbracket v \rrbracket \subseteq \llbracket w \rrbracket$ and thus conclude $\epsilon \in \llbracket \sigma \rrbracket$.

If $\sigma=(u \& v) \multimap w$. We have $\epsilon \in \llbracket(u \& v) \multimap w \rrbracket$ iff $\llbracket u \rrbracket \cap \llbracket v \rrbracket \subseteq \llbracket w \rrbracket$. If $\lfloor\Gamma\rfloor \in \llbracket u \rrbracket \cap \llbracket v \rrbracket$ then both $! \Sigma, \Gamma \vdash u$ and $! \Sigma, \Gamma \vdash v$ have a proof in Gill ${ }^{0}$. By rule $\langle(\&) \multimap\rangle$, the sequent $! \Sigma, \Gamma \vdash w$ has a proof in Gill ${ }^{0}$. Thus $\lfloor\Gamma\rfloor \in \llbracket w \rrbracket$. We have proved that $\llbracket u \rrbracket \cap \llbracket v \rrbracket \subseteq \llbracket w \rrbracket$ and we conclude $\epsilon \in \llbracket \sigma \rrbracket$.

Now let $\Sigma=\left\{\sigma_{1}, \ldots, \sigma_{k}\right\}$. Then for any $i \in[1, k]$ we have $\llbracket ! \sigma_{i} \rrbracket=\{\epsilon\} \cap \llbracket \sigma_{i} \rrbracket=\{\epsilon\}$. Consider $\Gamma=\left\lfloor a_{1}, \ldots, a_{p}\right\rfloor$ and let us suppose that the sequent $! \Sigma, \Gamma \vdash c$ of $\operatorname{ILL}_{-\infty, \&, !}^{0}$ is valid in every total deterministic trivial phase semantics interpretation. As a particular case, it is valid in the interpretation $(M, \circ, \epsilon, \llbracket \llbracket \rrbracket)$ and thus the inclusion

$$
\llbracket ! \sigma_{1} \rrbracket \circ \cdots \circ \llbracket ! \sigma_{k} \rrbracket \circ \llbracket a_{1} \rrbracket \circ \cdots \circ \llbracket a_{p} \rrbracket \subseteq \llbracket c \rrbracket
$$

holds. By rule $\langle\mathrm{Ax}\rangle$, for any $i \in[1, p]$ the sequent $! \Sigma, a_{i} \vdash a_{i}$ has a proof in Gill $^{0}$ and thus we have $\left\lfloor a_{i}\right\rfloor \in \llbracket a_{i} \rrbracket$. Also remember that for any $i \in[1, k]$, we have $\epsilon \in \llbracket ! \sigma_{i} \rrbracket$. So

$\lfloor\Gamma\rfloor \in\left\{\left\lfloor a_{1}, \ldots, a_{p}\right\rfloor\right\}=\epsilon \circ \cdots \circ \epsilon \circ\left\lfloor a_{1}\right\rfloor \circ \cdots \circ\left\lfloor a_{p}\right\rfloor \subseteq \llbracket c \rrbracket$ holds and we conclude that $! \Sigma, \Gamma \vdash c$ has a proof in Gill ${ }^{0}$.

Theorem 3.7. The system Gill ${ }^{0}$ and total deterministic trivial phase semantics are both sound and complete for the fragment $\mathrm{ILL}_{-\infty, \&, !}^{0}$. 
Proof. For a given sequent ! $\Sigma, \Gamma \vdash c$ of $\mathrm{ILL}_{-, \&, !}^{0}$, if it has a proof in Gill ${ }^{0}$ then, by Lemma 3.5, it has a proof in Sill. If $! \Sigma, \Gamma \vdash c$ is provable in Sill then, as a particular case of Theorem 2.9, it is valid in every total deterministic trivial phase semantics interpretation. Finally, if $! \Sigma, \Gamma \vdash c$ is valid in every total deterministic trivial phase semantics interpretation then, by Lemma 3.6, it is provable in Gill ${ }^{0}$.

\subsection{Encoding Minsky machines in ILL $_{-\circ, \&, !}^{0}$}

We propose an encoding of two counter Minsky machines in the fragment ILL ${ }_{-}^{0}, \&$ !! of ILL. Kanovich $[10,11]$ already proved that Minsky machines can be encoded into the $(!, \oplus)$-Horn fragment of ILL. In his encoding, the recovery of computations from proofs is done through some form of proof normalization and the $\oplus$ connective is used to simulate forking. Lafont later showed that the use of proof normalization can be avoided and replaced by a phase semantics argument $[12,14]$. In our encoding of Minsky machines in ILL $_{-, \&, !}^{0}$, the \& connective is used to simulate forking and we will show that a trivial phase semantics argument is sufficient to recover computability from provability.

Let $\mathrm{a}$ and $\mathrm{b}$ be two distinct counter symbols. A (deterministic) two counter Minsky machine is a pair $(l, \psi)$ where $l>0$ is a strictly positive natural number of instructions and

$$
\psi:[1, l] \longrightarrow \uplus \begin{aligned}
& \{+\} \times\{\mathrm{a}, \mathrm{b}\} \times[0, l] \\
& \{-\} \times\{\mathrm{a}, \mathrm{b}\} \times[0, l] \times[0, l]
\end{aligned}
$$

is a total map representing the list of instructions (here, $\uplus$ represents disjoint set union). Minsky machines instructions (incrementation, zero test/decrementation) are encoded as illustrated in the two following examples:

$$
\begin{gathered}
\psi(1)=(+, \mathrm{a}, 3) \rightsquigarrow 1: \mathrm{a}:=\mathrm{a}+1 ; \text { goto } 3 \\
\psi(2)=(-, \mathrm{b}, 4,5) \rightsquigarrow 2:\left\{\begin{array}{l}
\text { if } \mathrm{b}=0 \text { then goto } 4 \\
\text { else } \mathrm{b}:=\mathrm{b}-1 ; \operatorname{goto} 5
\end{array}\right.
\end{gathered}
$$

Given a two counter Minsky machine $\mathcal{M}=(l, \psi)$, we define the set $\mathcal{S}(\mathcal{M})$ of states of the machine by $\mathcal{S}(\mathcal{M})=$ $[0, l] \times \mathbb{N} \times \mathbb{N}$ and a (binary) transition relation between states $\rightarrow \mathcal{M} \subseteq \mathcal{S}(\mathcal{M}) \times \mathcal{S}(\mathcal{M})$. We define the following notations: $\overline{\mathrm{a}}=(1,0), \overline{\mathrm{b}}=(0,1),(m, n)_{\mathrm{a}}=m$ and $(m, n)_{\mathrm{b}}=n$. Then for any two states $(i, m, n)$ and $\left(i^{\prime}, m^{\prime}, n^{\prime}\right)$, we define the relation $(i, m, n) \rightarrow \mathcal{M}\left(i^{\prime}, m^{\prime}, n^{\prime}\right)$ by:

$$
\begin{aligned}
& \psi(i)=\left(+, x, i^{\prime}\right) \text { and }\left(m^{\prime}, n^{\prime}\right)=(m, n)+\bar{x} \\
& \text { or } \psi(i)=\left(-, x, i^{\prime}, k\right),(m, n)_{x}=0 \text { and }\left(m^{\prime}, n^{\prime}\right)=(m, n) \\
& \text { or } \psi(i)=\left(-, x, j, i^{\prime}\right),(m, n)_{x} \neq 0 \\
& \quad \text { and }\left(m^{\prime}, n^{\prime}\right)+\bar{x}=(m, n)
\end{aligned}
$$

holds for some $x \in\{\mathrm{a}, \mathrm{b}\}$ and $j, k \in[0, l]$. Notice that $(i, m, n) \rightarrow \mathcal{M}\left(i^{\prime}, m^{\prime}, n^{\prime}\right)$ does not hold if $i=0$ because $\psi(0)$ is not defined. Let $\rightarrow_{\mathcal{M}}^{\star}$ be the reflexive and transitive closure of the relation $\rightarrow \mathcal{M}$. We say that the machine $\mathcal{M}$ accepts the input $(m, n)$ if starting from the state $(1, m, n)$, there exists a sequence of transitions leading to the state $(0,0,0)$ and we define the set $\mathcal{A}(\mathcal{M})$ of accepted inputs:

$$
\mathcal{A}(\mathcal{M})=\left\{(m, n) \in \mathbb{N} \times \mathbb{N} \mid(1, m, n) \rightarrow_{\mathcal{M}}^{\star}(0,0,0)\right\}
$$

Theorem 3.8. There exists a two counter Minsky machine $\mathcal{M}$ for which the set $\mathcal{A}(\mathcal{M})$ is not recursive [18].

Let us consider the two counter symbols $a$ and $b$ as two (different) logical variables and let us choose two new variables $\mathrm{u}$ and $\mathrm{v}$ so that the set $\{\mathrm{a}, \mathrm{b}, \mathrm{u}, \mathrm{v}\}$ has cardinal four. Let us choose an infinite set $^{5}$ of new logical variables $\left\{\mathrm{q}_{i} \mid i \in \mathbb{N}\right\}$ such that $\mathrm{q}_{i} \neq \mathrm{q}_{j}$ unless $i=j$ and $\{\mathrm{a}, \mathrm{b}, \mathrm{u}, \mathrm{v}\} \cap\left\{\mathrm{q}_{i} \mid i \in \mathbb{N}\right\}=\emptyset$.

Let $\Sigma_{0}$ be the following multiset composed of five $(\multimap, \&)$-elementary formulae:

$$
\Sigma_{0}=\left\{\begin{array}{l}
\mathrm{a} \multimap(\mathrm{u} \multimap \mathrm{u}), \mathrm{b} \multimap(\mathrm{v} \multimap \mathrm{v}), \\
(\mathrm{a} \multimap \mathrm{a}) \multimap \mathrm{u},(\mathrm{a} \multimap \mathrm{a}) \multimap \mathrm{v},(\mathrm{a} \multimap \mathrm{a}) \multimap \mathrm{q}_{0}
\end{array}\right\}
$$

We define the two abbreviations $\underline{\mathrm{a}}=\mathrm{v}$ and $\underline{\mathrm{b}}=\mathrm{u}$. Given a Minsky machine $\mathcal{M}=(l, \psi)$, for $i \in[1, l]$, we define the multisets $\Sigma_{1}, \ldots, \Sigma_{l}$ of $(\multimap, \&)$-elementary formulae by:

$$
\begin{aligned}
\Sigma_{i} & =\left\{\left(x \multimap \mathrm{q}_{j}\right) \multimap \mathrm{q}_{i}\right\} \quad \text { when } \psi(i)=(+, x, j) \\
\text { and } \Sigma_{i} & =\left\{\left(\underline{x} \& \mathrm{q}_{j}\right) \multimap \mathrm{q}_{i}, x \multimap\left(\mathrm{q}_{k} \multimap \mathrm{q}_{i}\right)\right\} \\
& \text { when } \psi(i)=(-, x, j, k)
\end{aligned}
$$

Let $\Sigma_{\mathcal{M}}$ be the multiset $\Sigma_{\mathcal{M}}=\Sigma_{0}, \Sigma_{1}, \ldots, \Sigma_{l}$. Given a natural number $n$ and a logical variable $x \in\{\mathrm{a}, \mathrm{b}\}$, we define $x^{n}=x, x, \ldots, x$ as the multiset composed of $n$ occurrences of the variable $x$. Then, it is trivial to verify that for any natural numbers $m, n$ and any $i \in[0, l]$, the sequent ! $\Sigma_{\mathcal{M}}, \mathrm{a}^{m}, \mathrm{~b}^{n} \vdash \mathrm{q}_{i}$ belongs to the fragment ILL ${ }_{-, \&, !}^{0}$.

Theorem 3.9. For any two counter Minsky machine $\mathcal{M}$ and for any pair $m, n \in \mathbb{N}$, we have $(m, n) \in \mathcal{A}(\mathcal{M})$ if and only if the sequent $! \Sigma_{\mathcal{M}}, \mathrm{a}^{m}, \mathrm{~b}^{n} \vdash \mathrm{q}_{1}$ is provable in Gill ${ }^{0}$.

We detail the proof in the following discussion. Let us consider a fixed Minsky machine $\mathcal{M}=(l, \psi)$. Then we denote $\Sigma_{\mathcal{M}}($ resp. $\rightarrow \mathcal{M})$ simply by $\Sigma($ resp. $\rightarrow$ ). We decompose the proof in four main intermediate results.

Proposition 3.10. For any $m, n \in \mathbb{N}$, the sequents $! \Sigma$, $\mathrm{a}^{m} \vdash$ $\mathrm{u}$ and $! \Sigma, \mathrm{b}^{n} \vdash \mathrm{v}$ are provable in Gill ${ }^{0}$.

Proof. We prove the case with $\mathrm{a} / \mathrm{u}$. The case of $\mathrm{b} / \mathrm{v}$ is

\footnotetext{
${ }^{5}$ For our particular purpose, we only need as many $\mathrm{q}_{i}$ 's as there are instructions in the Minsky machine obtained from Theorem 3.8.
} 
similar (see [16], Appendix D). Here is a suitable proof tree:

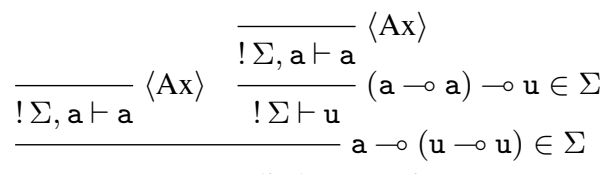

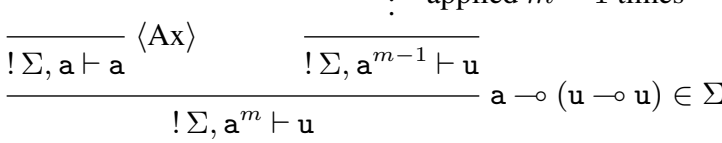

In fact, this is the only possible proof tree but the demonstration of this uniqueness result is left to the reader.

Lemma 3.11. For any $r, m, n \in \mathbb{N}$ and any $i \in[0, l]$, if $(i, m, n) \rightarrow^{r}(0,0,0)$ then the sequent $! \Sigma, \mathrm{a}^{m}, \mathrm{~b}^{n} \vdash \mathrm{q}_{i}$ is provable in Gill $^{0}$.

Proof. We proceed by induction on $r$. If $r=0$ then we have $(i, m, n)=(0,0,0)$. The sequent $! \Sigma \vdash \mathrm{q}_{0}$ has the following proof tree:

$$
\frac{\overline{! \Sigma, \mathrm{a} \vdash \mathrm{a}}\langle A \mathrm{x}\rangle}{\frac{! \Sigma \vdash \mathrm{q}_{0}}{\mathrm{a}}(\mathrm{a} \multimap \mathrm{a}) \multimap \mathrm{q}_{0} \in \Sigma}
$$

Let us now consider a transition sequence $(i, m, n) \rightarrow$ $\left(i^{\prime}, m^{\prime}, n^{\prime}\right) \rightarrow^{r}(0,0,0)$ of length $r+1$. By induction hypothesis, let $P$ be a proof tree for the sequent $! \Sigma, \mathrm{a}^{m^{\prime}}, \mathrm{b}^{n^{\prime}} \vdash$ $\mathrm{q}_{i^{\prime}}$. We consider the three cases for $(i, m, n) \rightarrow\left(i^{\prime}, m^{\prime}, n^{\prime}\right)$.

If $\psi(i)=\left(+, x, i^{\prime}\right)$ and $\left(m^{\prime}, n^{\prime}\right)=(m, n)+\bar{x}$. Without loss of generality, we consider the case $x=$ a (the case $x=\mathrm{b}$ is similar). Then $m^{\prime}=m+1$ and $n^{\prime}=n$. We provide the following proof tree for $! \Sigma, \mathrm{a}^{m}, \mathrm{~b}^{n} \vdash \mathrm{q}_{i}$ :

$$
\frac{P}{\frac{! \Sigma, \mathrm{a}^{m}, \mathrm{~b}^{n}, \mathrm{a} \vdash \mathrm{q}_{i^{\prime}}}{! \Sigma, \mathrm{a}^{m}, \mathrm{~b}^{n} \vdash \mathrm{q}_{i}}\left(\mathrm{a} \multimap \mathrm{q}_{i^{\prime}}\right) \multimap \mathrm{q}_{i} \in \Sigma}
$$

If $\psi(i)=\left(-, x, i^{\prime}, k\right),(m, n)_{x}=0$ and $\left(m^{\prime}, n^{\prime}\right)=$ $(m, n)$. We consider the case $x=$ a without loss of generality. Then $m=m^{\prime}=0$ and $n=n^{\prime}$. Let $Q$ be a proof tree for $! \Sigma, \mathrm{b}^{n} \vdash \mathrm{v}$ according to Proposition 3.10. We provide the following proof tree for $! \Sigma, \mathrm{b}^{n} \vdash \mathrm{q}_{i}$ :

$$
\frac{\frac{Q}{! \Sigma, \mathrm{b}^{n} \vdash \mathrm{v}} \frac{P}{! \Sigma, \mathrm{b}^{n} \vdash \mathrm{q}_{i^{\prime}}}}{! \Sigma, \mathrm{b}^{n} \vdash \mathrm{q}_{i}}\left(\mathrm{v} \& \mathrm{q}_{i^{\prime}}\right) \multimap \mathrm{q}_{i} \in \Sigma
$$

If $\psi(i)=\left(-, x, j, i^{\prime}\right),(m, n)_{x} \neq 0$ and $\left(m^{\prime}, n^{\prime}\right)+\bar{x}=$ $(m, n)$. We consider the case $x=$ a without loss of generality. Then $m=m^{\prime}+1$ and $n=n^{\prime}$. We provide the following proof tree for ! $\Sigma, \mathrm{a}, \mathrm{a}^{m^{\prime}}, \mathrm{b}^{n^{\prime}} \vdash \mathrm{q}_{i}$ :

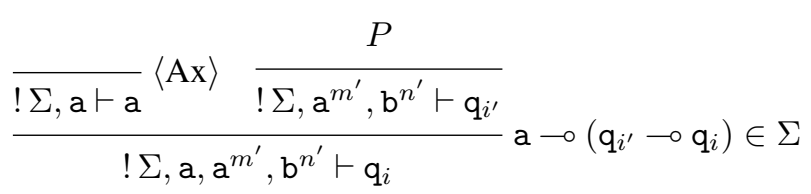

In any case we obtain a proof tree for $! \Sigma, \mathrm{a}^{m}, \mathrm{~b}^{n} \vdash \mathrm{q}_{i}$ which fulfills the induction step. ${ }^{6}$

We point out that the form $(\&) \multimap$ is used to encode forking in a way similar Kanovich does with $\oplus$ (see [11]).

Let us now consider the following total deterministic trivial phase semantics interpretation. The free commutative monoid over two elements is $(\mathbb{N} \times \mathbb{N},+,(0,0))$. We define $x \circ y=\{x+y\}$ and $(\mathbb{N} \times \mathbb{N}, \circ,(0,0))$ is thus a total deterministic monoid. Every subset of $\mathbb{N} \times \mathbb{N}$ is closed in trivial phase semantics and we define

$$
\begin{aligned}
& \llbracket \mathrm{a} \rrbracket=\{(1,0)=\overline{\mathrm{a}}\} \quad \llbracket \mathrm{u} \rrbracket=\mathbb{N} \times\{0\} \\
& \llbracket \mathrm{b} \rrbracket=\{(0,1)=\overline{\mathrm{b}}\} \quad \llbracket \mathrm{v} \rrbracket=\{0\} \times \mathbb{N} \\
& \llbracket \mathrm{q}_{i} \rrbracket=\left\{(m, n) \in \mathbb{N} \times \mathbb{N} \mid(i, m, n) \rightarrow^{\star}(0,0,0)\right\}
\end{aligned}
$$

It is crucial that variables $a, b, u, v, q_{0}, q_{1}, \ldots, q_{l}$ were chosen distinct from one another for this definition to be valid. Let us now consider the trivial phase semantics interpretation of the compound formulae of $\Sigma$.

Proposition 3.12. For any $\sigma \in \Sigma$, $\llbracket ! \sigma \rrbracket=\{(0,0)\}$ holds.

Proof. As the identity $\llbracket ! \sigma \rrbracket=\{(0,0)\} \cap \llbracket \sigma \rrbracket$ holds in the trivial phase semantics interpretation, it is necessary and sufficient to prove that $(0,0) \in \llbracket \sigma \rrbracket$ holds for any $\sigma \in \Sigma$.

First let us prove that $\llbracket \mathrm{a} \multimap \mathrm{a} \rrbracket=\{(0,0)\}$. Indeed, $(m, n) \in \llbracket \mathrm{a} \multimap \mathrm{a} \rrbracket$ iff $(m, n) \circ \llbracket \mathrm{a} \rrbracket \subseteq \llbracket \mathrm{a} \rrbracket$ iff $(m, n) \circ$ $\{(1,0)\} \subseteq\{(1,0)\}$ iff $\{(m+1, n)\} \subseteq\{(1,0)\}$ iff $(m, n)=(0,0)$. Then $\llbracket(\mathrm{a} \multimap \mathrm{a}) \multimap x \rrbracket=\{(0,0)\} \multimap \llbracket x \rrbracket=$ [x] for any variable $x$, in particular for $x \in\left\{\mathrm{u}, \mathrm{v}, \mathrm{q}_{0}\right\}$. Also $(m, n) \in \llbracket \mathrm{a} \multimap(\mathrm{u} \multimap \mathrm{u}) \rrbracket$ iff $(m, n) \circ\{(1,0)\} \circ \mathbb{N} \times\{0\} \subseteq$ $\mathbb{N} \times\{0\}$ iff $n=0$. Thus $\llbracket \mathrm{a} \multimap(\mathrm{u} \multimap \mathrm{u}) \rrbracket=\mathbb{N} \times\{0\}$. By a similar argument, we get $\llbracket \mathrm{b} \multimap(\mathrm{v} \multimap \mathrm{v}) \rrbracket=\{0\} \times \mathbb{N}$. So for any formula $\sigma \in \Sigma_{0}$, we have $(0,0) \in \llbracket \sigma \rrbracket$.

Let us consider the formulae in $\Sigma_{i}$ for $i \in[1, l]$. Let us prove that the relation $(0,0) \in \llbracket \sigma \rrbracket$ holds for any $\sigma \in \Sigma_{i}$.

If $\psi(i)=(+, x, j)$. Let us prove $(0,0) \in \llbracket\left(x \multimap \mathrm{q}_{j}\right) \multimap$ $\mathrm{q}_{i} \rrbracket$, i.e., $\llbracket x \multimap \mathrm{q}_{j} \rrbracket \subseteq \llbracket \mathrm{q}_{i} \rrbracket$. Let us consider $(m, n) \in \llbracket x \multimap$ $\mathrm{q}_{j} \rrbracket$. Then $\{(m, n)+\bar{x}\}=\{(m, n)\} \circ \llbracket x \rrbracket \subseteq \llbracket \mathrm{q}_{j} \rrbracket$ and thus $\left(m^{\prime}, n^{\prime}\right)=(m, n)+\bar{x} \in \llbracket\left[\mathrm{q}_{j} \rrbracket\right.$. Thus we have $(i, m, n) \rightarrow$ $\left(j, m^{\prime}, n^{\prime}\right) \rightarrow^{\star}(0,0,0)$. We conclude $(m, n) \in \llbracket q_{i} \rrbracket$.

If $\psi(i)=(-, x, j, k)$. Let us first prove that $(0,0) \in$ $\llbracket\left(\underline{x} \& \mathrm{q}_{j}\right) \multimap \mathrm{q}_{i} \rrbracket$, i.e., $\llbracket \underline{x} \rrbracket \cap \llbracket \mathrm{q}_{j} \rrbracket \subseteq \llbracket \mathrm{q}_{i} \rrbracket$. Let us consider $(m, n) \in \llbracket \underline{x} \rrbracket \cap \llbracket \mathrm{q}_{j} \rrbracket$. Then $(m, n)_{x}=0$ and $(j, m, n) \rightarrow^{\star}(0,0,0)$. Thus $(i, m, n) \rightarrow(j, m, n) \rightarrow^{\star}$ $(0,0,0)$ and $(m, n) \in \llbracket \mathrm{q}_{i} \rrbracket$. Hence $\llbracket \underline{x} \rrbracket \cap \llbracket \mathrm{q}_{j} \rrbracket \subseteq \llbracket \mathrm{q}_{i} \rrbracket$ holds. Let us finally prove that $(0,0) \in \llbracket x \multimap\left(\mathrm{q}_{k} \multimap \mathrm{q}_{i}\right) \rrbracket$, i.e., $\llbracket x \rrbracket \circ \llbracket \mathrm{q}_{k} \rrbracket \subseteq \llbracket \mathrm{q}_{i} \rrbracket$. As $\llbracket x \rrbracket=\{\bar{x}\}$ for $x \in\{\mathrm{a}, \mathrm{b}\}$, let us choose an arbitrary pair $\left(m^{\prime}, n^{\prime}\right) \in \llbracket \mathrm{q}_{k} \rrbracket$ and define $(m, n)=\bar{x}+\left(m^{\prime}, n^{\prime}\right)$. Then $(m, n)_{x}=1+\left(m^{\prime}, n^{\prime}\right)_{x} \neq 0$

\footnotetext{
${ }^{6}$ Again, but this is left to the reader, it can be demonstrated that the proof tree recursively built from the transition sequence $(i, m, n) \rightarrow^{r}$ $(0,0,0)$ is the unique proof tree for the sequent $! \Sigma, \mathrm{a}^{m}, \mathrm{~b}^{n} \vdash \mathrm{q}_{i}$.
} 
and $(i, m, n) \rightarrow\left(k, m^{\prime}, n^{\prime}\right) \rightarrow^{\star}(0,0,0)$. We obtain $(m, n) \in \llbracket \mathrm{q}_{i} \rrbracket$ and thus conclude $\bar{x}+\left(m^{\prime}, n^{\prime}\right) \in \llbracket \mathrm{q}_{i} \rrbracket$. Hence, for any $\left(m^{\prime}, n^{\prime}\right) \in \llbracket \mathrm{q}_{k} \rrbracket$ we get $\llbracket x \rrbracket \circ\left(m^{\prime}, n^{\prime}\right) \subseteq$ $\llbracket \mathrm{q}_{i} \rrbracket$. Then $\llbracket x \rrbracket \circ \llbracket \mathrm{q}_{k} \rrbracket \subseteq \llbracket \mathrm{q}_{i} \rrbracket$ holds.

As a consequence, for any $\sigma \in \Sigma$, we obtain $(0,0) \in$ $\llbracket \sigma \rrbracket$. The identity $\llbracket ! \sigma \rrbracket=\{(0,0)\}$ holds for any $\sigma \in \Sigma$.

Lemma 3.13. For any $m, n \in \mathbb{N}$, if $! \Sigma, \mathrm{a}^{m}, \mathrm{~b}^{n} \vdash \mathrm{q}_{1}$ is provable in $\mathrm{Gill}^{0}$ then $(m, n) \in \mathcal{A}(\mathcal{M})$ holds.

Proof. Let $\Sigma=\left\{\sigma_{1}, \ldots, \sigma_{r}\right\}$. We suppose that the sequent $! \Sigma, \mathrm{a}^{m}, \mathrm{~b}^{n} \vdash \mathrm{q}_{1}$ has a proof in Gill ${ }^{0}$. By the soundness part of Theorem 3.7, in our particular total deterministic trivial phase semantics interpretation, we have

$\llbracket ! \sigma_{1} \rrbracket \circ \cdots \circ \llbracket ! \sigma_{r} \rrbracket \circ \llbracket \mathrm{a} \rrbracket \circ \cdots \circ \llbracket \mathrm{a} \rrbracket \circ \llbracket \mathrm{b} \rrbracket \circ \cdots \circ \llbracket \mathrm{b} \rrbracket \subseteq \llbracket \mathrm{q}_{1} \rrbracket$

where a occurs $m$ times and b occurs $n$ times. By Proposition 3.12, we deduce $(m, n)=r .(0,0)+m .(1,0)+$ $n .(0,1) \in \llbracket \mathrm{q}_{1} \rrbracket$ and thus $(1, m, n) \rightarrow^{\star}(0,0,0)$ holds.

This concludes the proof of Theorem 3.9 as direct consequence of Lemma 3.11 and Lemma 3.13.

The reader may have noticed that more than the simple encoding of computability with provability, we can even show that computations and proofs match one to one. Even though this result is not necessary to our argumentation, this suggests that the system Gill $^{0}$ is a natural choice to illustrate the relations between Minsky machines and linear logic, and may be more straightforward than the $(!, \oplus)$-Horn fragment [11]. Whereas the decidability of $s-I_{M E L L} \multimap$ is still unclear (but nevertheless known to be equivalent to the decidability of MELL [4]), we have proved that the simple addition of the form $(\&) \multimap$ to $S-$ IMELL $_{0}^{-}$is sufficient to encode forking and thus, computations of Minsky machines.

Theorem 3.14. Validity in ILL ${ }_{-, \&, !}^{0}$ is undecidable.

Proof. By Theorem 3.8, let $\mathcal{M}$ be a two counter Minsky machine s.t. $\mathcal{A}(\mathcal{M})$ is not recursive. Compute $\Sigma_{\mathcal{M}}$. If there is an algorithm that discriminates between provable and unprovable sequents of ILL ${ }_{-}^{0} \&$ ! , use it to decide $\mathcal{A}(\mathcal{M})=$ $\left\{(m, n) \in \mathbb{N} \times \mathbb{N} \mid ! \Sigma, \mathrm{a}^{m}, \mathrm{~b}^{n} \vdash \mathrm{q}_{1}\right.$ is provable in Gill $\left.{ }^{0}\right\}$ This identity is a direct consequence of Theorem 3.9. Thus $\mathcal{A}(\mathcal{M})$ would be recursive. We obtain a contradiction.

Acknowledgments. The authors dedicate this work and the resulting paper to the memory of Pr. Noëlle Carbonell.

\section{References}

[1] J. Brotherston. A cut free proof theory for Boolean BI. Technical Report DTR09-13, Imperial College London, 2009.

[2] J. Brotherston and C. Calcagno. Classical BI: a logic for reasoning about dualising resources. In Z. Shao and B. C. Pierce, editors, POPL, pages 328-339. ACM, 2009.
[3] C. Calcagno, L. Cardelli, and A. Gordon. Deciding Validity in a Spatial Logic for Trees. J. Funct. Program., 15(4):543$572,2005$.

[4] P. de Groote, B. Guillaume, and S. Salvati. Vector addition tree automata. In LICS'04, pages 64-73. IEEE, 2004.

[5] D. Galmiche and D. Larchey-Wendling. Expressivity properties of Boolean BI through relational models. In FSTTCS, volume 4337 of $L N C S$, pages 357-368. Springer, 2006.

[6] D. Galmiche, D. Méry, and D. Pym. The semantics of $\mathrm{BI}$ and resource tableaux. Math. Struct. in Comp. Science, 15(6): 1033-1088, 2005.

[7] S. Ghilardi and G. Meloni. Modal logics with n-ary connectives. Zeitschr. f. math. Logik und Grundlagen d. Math, 36:193-215, 1990.

[8] J.-Y. Girard. Linear logic. TCS, 50(1):1-102, 1987.

[9] S. Ishtiaq and P. O'Hearn. BI as an Assertion Language for Mutable Data Structures. In POPL, pages 14-26, 2001.

[10] M. Kanovich. Linear Logic as a Logic of Computations. Annals of Pure and Applied Logic, 67(1-3):183-212, 1994.

[11] M. Kanovich. The direct simulation of Minsky machines in linear logic. In Advances in Linear Logic, volume 222, chapter 2, pages 123-145. Cambridge U. Press, 1995.

[12] Y. Lafont. The undecidability of second order linear logic without exponentials. J. Symb. Logic, 61(2):541-548, 1996.

[13] Y. Lafont. The finite model property for various fragments of linear logic. J. Symb. Logic, 62(4):1202-1208, 1997.

[14] Y. Lafont and A. Scedrov. The undecidability of second order multiplicative linear logic. Information and Computation, 125(1):46-51, 1996.

[15] D. Larchey-Wendling and D. Galmiche. Exploring the relation between Intui. BI and Boolean BI: an unexpected embedding. Math. Struct. in Comp. Science, 19(3):435-500, 2009.

[16] D. Larchey-Wendling and D. Galmiche. The Undecidability of Boolean BI through Phase Semantics (full version). Available at http://www. loria.fr/ larchey.

[17] P. Lincoln, J. Mitchell, A. Scedrov, and N. Shankar. Decision problems for propositional linear logic. In FOCS, volume 2, pages 662-671. IEEE, 1990.

[18] M. Minsky. Recursive unsolvability of Post's problem of 'tag' and other topics in the theory of Turing machines. Annals of Mathematics, 74(3):437-455, 1961.

[19] P. O'Hearn and D. Pym. The logic of bunched implications. Bulletin of Symbolic Logic, 5(2):215-244, 1999.

[20] M. Okada. A uniform semantic proof for cut-elimination and completeness of various first and higher order logics. TCS, 281(1-2):471-498, 2002.

[21] D. Pym. The Semantics and Proof Theory of the Logic of Bunched Implications. Kluwer Academic Publishers, 2002.

[22] G. Restall. Displaying and Deciding Substructural Logics 1: Logics with Contraposition. Journal of Philosophical Logic, 27:179-216, 1998.

[23] A. Troelstra. Lectures on Linear Logic, volume 29 of Lecture Notes. CSLI, Stanford, California, 1992.

[24] D. Yetter. Quantales and (Noncommutative) Linear Logic. J. Symb. Logic, 55(1):41-64, 1990. 


\section{A. A witness for $\mathrm{BBI}_{\mathrm{TD}} \not \subset \mathbf{B B I _ { \mathrm { PD } }}$}

Proposition 2.7 The formula $(\neg \mid \rightarrow \perp) \rightarrow \mid$ is valid in every total deterministic monoid. There exists a partial deterministic monoid which is a counter-model to $(\neg \mathrm{I} \rightarrow \perp) \rightarrow \mathrm{I}$.

Proof. First the counter-model. Consider the following partial deterministic monoid $(\{\epsilon, \mathrm{x}\}, \circ, \epsilon)$ where $\mathrm{x} \circ \mathrm{x}=\emptyset$ and $\epsilon \circ \alpha=\alpha \circ \epsilon=\{\alpha\}$ for any $\alpha \in\{\epsilon, \mathrm{x}\}$. Then $\mathrm{x} \neq \epsilon$ and thus $\mathrm{x} \nVdash \mathrm{I}$. Let us prove that $\mathrm{x} \Vdash \neg \mathrm{I} * \perp$. Let $a, b$ such that $b \in \mathrm{x} \circ a$ and $a \Vdash \neg$. Then $a \neq \epsilon$ and thus $a=\mathrm{x}$. Then $\mathrm{x} \circ a=\mathrm{x} \circ \mathrm{x}=\emptyset$. We get a contradiction with $b \in \mathrm{x} \circ a$. From this contradiction, we deduce $b \Vdash \perp$. Hence, $\mathrm{x} \Vdash \neg \mathrm{I} * \perp$ and we conclude $\mathrm{x} \nVdash(\neg \mathrm{I} * \perp) \rightarrow \mathrm{I}$ and we have the counter-model.

Now let us prove that $(\neg \mid * \perp) \rightarrow \mid$ is valid in every total deterministic monoid. Let $(M, \circ, \epsilon)$ be a total deterministic monoid. Let us choose $a \in M$. There are two cases. Either $a=\epsilon$ or $a \neq \epsilon$. In the case $a=\epsilon$, we obviously have $a \Vdash(\neg \mathbf{I} * \perp) \rightarrow \mathrm{I}$. In the case $a \neq \epsilon$, let us prove $a \nVdash \neg \mathrm{I} * \perp$. Suppose $a \Vdash \neg \mathrm{l} \rightarrow \perp$. As $a \neq \epsilon$ we have $a \Vdash \neg$ l. Also $a \circ a$ is not empty because $\circ$ is total. Let $b \in a \circ a$. As $a \Vdash \neg \mathrm{I} * \perp, b \in a \circ a$ and $a \Vdash \neg$, we must have $b \Vdash \perp$ which is impossible. Hence $a \nVdash \neg I * \perp$ and we conclude that $a \Vdash(\neg \mid+\perp) \rightarrow \mid$ holds also in the case $a \neq \epsilon$.

\section{B. Soundness of non-deterministic phase se- mantics for ILL}

Theorem 2.9 Let $\mathcal{M}=\left(M, \circ, \epsilon,(\cdot)^{\triangleright}, K\right)$ be a non-deterministic intuitionistic phase space and $\llbracket \cdot \rrbracket: \operatorname{Var} \longrightarrow \mathcal{M}^{\diamond}$ be an interpretation of logical variables. If the sequent $A_{1}, \ldots, A_{k} \vdash B$ has a proof in Sill, then the inclusion $\llbracket A_{1} \rrbracket \circ \cdots \circ \llbracket A_{k} \rrbracket \subseteq \llbracket B \rrbracket$ holds.

Proof. It could be done by induction on ILL proof trees but we rather use the algebraic semantic characterization of ILL of [23]. We prove that

$$
\left(\mathcal{M}^{\diamond}, \cap,(\cdot \cup \cdot)^{\diamond}, \emptyset^{\diamond}, \multimap,(\cdot \circ \cdot)^{\diamond},\{\epsilon\}^{\diamond},(K \cap \cdot)^{\diamond}\right)
$$

is an IL-algebra with storage operator (where $\multimap$ is defined by $X \multimap Y=\{k \in M \mid k \circ X \subseteq Y\})$.

First, it is obvious that $\left(\mathcal{M}^{\diamond}, \cap,(\cdot \cup \cdot)^{\triangleright}, \emptyset^{\diamond}\right)$ is a complete lattice with bottom $\emptyset^{\diamond}$. This is the same proof as in the usual (monoidal) case because the (non-deterministic) monoidal structure does not play any role in this part of the proof. The principal argument is that $(\cdot)^{\diamond}$ is a closure operator in $\mathcal{P}(M)$.

Let us prove that $\left(\mathcal{M}^{\diamond},(\cdot \circ \cdot)^{\diamond},\{\epsilon\}^{\diamond}\right)$ is a commutative monoid. Obviously the set $\mathcal{M}^{\diamond}$ is stable under the operator $(\cdot \circ \cdot)^{\diamond}$ which thus induces a binary operation on $\mathcal{M}^{\diamond}$. By stability, we obtain the inclusion $\{\epsilon\}^{\diamond} \circ X^{\diamond} \subseteq(\{\epsilon\} \circ X)^{\diamond}=$
$X^{\diamond}$ and we deduce that for any closed subset $X$ (i.e. $X=$ $\left.X^{\diamond}\right)$, we have $\left(\epsilon^{\diamond} \circ X\right)^{\diamond} \subseteq X$. Also $X=\{\epsilon\} \circ X \subseteq$ $\{\epsilon\}^{\diamond} \circ X \subseteq\left(\{\epsilon\}^{\diamond} \circ X\right)^{\diamond}$ by monotonicity of $\circ$ and $(\cdot)^{\diamond}$. Thus $\left(\epsilon^{\diamond} \circ X\right)^{\diamond}=X$ for any closed subset $X \in \mathcal{M}^{\diamond}$ and thus $\{\epsilon\}^{\diamond}$ is a (left) unit for $(\cdot \circ \cdot)^{\diamond}$. Then, it is obvious that $(\cdot \circ \cdot)^{\diamond}$ is a commutative operation because $\circ$ is itself commutative. We deduce that $\{\epsilon\}^{\diamond}$ is a unit for $(\cdot \circ \cdot)^{\diamond}$.

Let us prove that $(\cdot \circ \cdot)^{\diamond}$ is associative. Let $A, B, C \in$ $\mathcal{M}^{\diamond}$. Then, by stability of $(\cdot)^{\diamond}$, we have $A \circ(B \circ C)^{\diamond} \subseteq$ $A^{\diamond} \circ(B \circ C)^{\diamond} \subseteq(A \circ(B \circ C))^{\diamond}=(A \circ B \circ C)^{\diamond}$. Thus $\left(A \circ(B \circ C)^{\diamond}\right)^{\diamond} \subseteq(A \circ B \circ C)^{\diamond}$ holds. As $A \circ B \circ C=$ $A \circ(B \circ C) \subseteq A \circ(B \circ C)^{\diamond} \subseteq\left(A \circ(B \circ C)^{\diamond}\right)^{\diamond}$, we deduce $(A \circ B \circ C)^{\diamond} \subseteq\left(A \circ(B \circ C)^{\diamond}\right)^{\diamond}$. By double inclusion, we conclude that $(A \circ B \circ C)^{\diamond}=\left(A \circ(B \circ C)^{\diamond}\right)^{\diamond}$. Associativity follows from this last identity and associativity/commutativity of $\circ$ on $\mathcal{P}(M)$.

It is obvious that $(\cdot \circ \cdot)^{\diamond}$ is monotonic in both parameters because it is obtained by composition of two monotonic operators, namely $\circ$ and $(\cdot)^{\diamond}$. Let us now prove that $\multimap$ is a right-adjoint $(\cdot \circ \cdot)^{\diamond}$. First, $X \multimap Y$ is closed as soon as $Y$ is closed and $X \multimap Y^{\diamond}=X^{\diamond} \multimap Y^{\diamond}$ holds for any $X, Y \in \mathcal{P}(M)$ just as in the usual (monoidal) case. Now let $A, B, C \in \mathcal{M}^{\diamond}$. We have $(A \circ B)^{\diamond} \subseteq C$ iff $A \circ B \subseteq C$ iff $A \subseteq B \multimap C$. Thus $\multimap$ is indeed right-adjoint to $(\cdot \circ \cdot)^{\diamond}$. The fact that $\multimap$ is contra-variant w.r.t. its first operand and co-variant w.r.t. its second operand is deducible from the monotonicity of $\circ$ and the fact that $\multimap$ is right adjoint to $\circ$.

We finish by proving that $X \mapsto(K \cap X)^{\diamond}$ is a modality. First, for any $X \in \mathcal{M}^{\diamond}$, as $K \cap X \subseteq X=X^{\diamond}$, we obtain $(K \cap X)^{\diamond} \subseteq X$. Then for $X, Y \in \mathcal{M}^{\diamond}$, if we suppose that $(K \cap \bar{Y})^{\diamond} \subseteq X$, then $K \cap Y \subseteq X$ and thus $K \cap Y \subseteq K \cap X$. Thus we obtain $(K \cap Y)^{\diamond} \subseteq$ $(K \cap X)^{\diamond}$. Then, as $\epsilon \in K \subseteq\{\epsilon\}^{\diamond}$, we deduce $\{\epsilon\}^{\diamond} \subseteq K^{\diamond}=(K \cap M)^{\diamond}{ }^{7}$ The last condition to check is $\left((K \cap X)^{\diamond} \circ(K \cap Y)^{\diamond}\right)^{\diamond}=(K \cap X \cap Y)^{\diamond}$ for any $X, Y \in \mathcal{M}^{\diamond}$. First we have $(K \cap X)^{\diamond} \circ(K \cap Y)^{\diamond} \subseteq$ $((K \cap X) \circ(K \cap Y))^{\diamond}$. As $K \subseteq\{\epsilon\}^{\diamond}$, we have $(K \cap$ $X) \circ(K \cap Y) \subseteq\{\epsilon\}^{\diamond} \circ Y \subseteq Y^{\diamond}=Y$. We also have $(K \cap X) \circ(K \cap Y) \subseteq X$. As $K \circ K \subseteq K$ we have $(K \cap X) \circ$ $(K \cap Y) \subseteq K$ and hence, we deduce $(K \cap X) \circ(K \cap Y) \subseteq$ $K \cap X \cap Y$. Using stability, we compute $(K \cap X)^{\diamond} \circ$ $(K \cap Y)^{\diamond} \subseteq((K \cap X) \circ(K \cap Y))^{\diamond} \subseteq(K \cap X \cap Y)^{\diamond}$ and thus $\left((K \cap X)^{\diamond} \circ(K \cap Y)^{\diamond}\right)^{\diamond} \subseteq(K \cap X \cap Y)^{\diamond}$. Now let us prove the reverse inclusion. Let $z \in K \cap X \cap Y$. As $z \in K$ then $z \in J$ and we have $z \in(z \circ z)^{\diamond} \subseteq$ $((K \cap X) \circ(K \cap Y))^{\diamond} \subseteq\left((K \cap X)^{\diamond} \circ(K \cap Y)^{\diamond}\right)^{\diamond}$. Hence, $K \cap X \cap Y \subseteq\left((\bar{K} \cap X)^{\diamond} \circ(K \cap Y)^{\diamond}\right)^{\diamond}$ and we deduce $(K \cap X \cap Y)^{\diamond} \subseteq\left((K \cap X)^{\diamond} \circ(K \cap Y)^{\diamond}\right)^{\diamond}$.

We can then apply Theorem 8.21 (page 80) from [23]. If $A_{1}, \ldots, A_{k} \vdash B$ has a proof in ILL, then the inclusion $\llbracket A_{1}, \ldots, A_{k} \rrbracket \subseteq \llbracket B \rrbracket$ holds. It is obvious to prove that $\llbracket A_{1} \rrbracket \circ \cdots \circ \llbracket A_{k} \rrbracket \subseteq \llbracket A_{1}, \ldots, A_{k} \rrbracket$ by induction on $k$ for

\footnotetext{
${ }^{7}$ Recall the identity $\emptyset^{\diamond} \multimap \emptyset^{\diamond}=\emptyset \multimap \emptyset^{\diamond}=M$.
} 
example. So we deduce $\llbracket A_{1} \rrbracket \circ \cdots \circ \llbracket A_{k} \rrbracket \subseteq \llbracket B \rrbracket$.

\section{The soundness of Gill ${ }^{0}$}

Lemma 3.5 Every proof of a sequent in Gill ${ }^{0}$ can be transformed into a proof of the same sequent which uses only the rules $\langle\mathrm{id}\rangle,\langle\mathrm{w}\rangle,\langle\mathrm{c}\rangle,\left\langle\rightarrow_{L}\right\rangle,\left\langle\multimap_{R}\right\rangle,\left\langle!_{L}\right\rangle$ and $\left\langle \&_{R}\right\rangle$ of Sill.

Proof. We proceed by induction on the proofs in Gill ${ }^{0}$ and by case analysis, depending on the last rule applied. Let $n$ be the cardinal of the multiset $\Sigma$. For each rule of Gill ${ }^{0}$, we propose the corresponding (open) proof tree in Sill:

- Case of rule $\langle\mathrm{Ax}\rangle$.

$$
\begin{aligned}
& \overline{a \vdash a}\langle\mathrm{id}\rangle \\
& \frac{\mathrm{a}}{\vdots \quad} \quad \text { applied } n \text { times } \\
& \frac{\mathrm{l}}{! \Sigma, a \vdash a}\langle\mathrm{w}\rangle
\end{aligned}
$$

- Case of rule $\langle\multimap\rangle$ :

$$
\begin{gathered}
\frac{! \Sigma, \Gamma \vdash a \quad \overline{b \vdash b}}{! \Sigma, \Gamma, a \multimap b \vdash b}\left\langle\multimap_{L}\right\rangle \\
\frac{! \Sigma, \Gamma, !(a \multimap b) \vdash b}{! \Sigma, \Gamma \vdash b}\left\langle!_{L}\right\rangle \\
\text { c }\rangle
\end{gathered}
$$

- Case of rule $\langle(\multimap)-\rangle$ :

$$
\begin{aligned}
& \frac{\frac{! \Sigma, \Gamma, a \vdash b}{! \Sigma, \Gamma \vdash a \multimap b}\langle\multimap R}{\frac{!}{c \vdash c}}\langle\mathrm{id}\rangle \\
& \frac{\frac{! \Sigma, \Gamma,(a \multimap b) \multimap c \vdash c}{! \Sigma, \Gamma, !((a \multimap b) \multimap c) \vdash c}}{! \Sigma, \Gamma \vdash c}\left\langle!_{L}\right\rangle \\
& \left.\frac{\mathrm{c}\rangle}{L}\right\rangle
\end{aligned}
$$

- Case of rule $\langle\multimap(\multimap)\rangle$ :

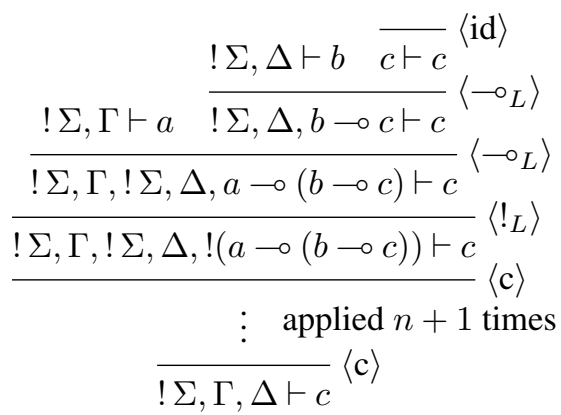

- Case of rule $\langle(\&) \multimap\rangle$ :

$$
\begin{gathered}
\frac{! \Sigma, \Gamma \vdash a \quad ! \Sigma, \Gamma \vdash b}{! \Sigma, \Gamma \vdash a \& b}\left\langle \&_{R}\right\rangle \quad \overline{c \vdash c}\langle\mathrm{id}\rangle \\
\frac{\frac{! \Sigma, \Gamma,(a \& b) \multimap c \vdash c}{! \Sigma, \Gamma, !((a \& b) \multimap c) \vdash c}}{! \Sigma, \Gamma \vdash c}\left\langle!_{L}\right\rangle \\
\left.\frac{\mathrm{c}}{L}\right\rangle
\end{gathered}
$$

\section{The soundness of the encoding}

Proposition 3.10 For any $m, n \in \mathbb{N}$, the sequents $! \Sigma, \mathrm{a}^{m} \vdash$ $\mathrm{u}$ and $! \Sigma, \mathrm{b}^{n} \vdash \mathrm{v}$ are provable in Gill ${ }^{0}$.

Proof. We prove the remaining case with $\mathrm{b}$ and v. Here is a suitable proof tree:

$$
\begin{aligned}
& \frac{\overline{! \Sigma, \mathrm{b} \vdash \mathrm{b}}\langle\mathrm{Ax}\rangle \frac{\overline{! \Sigma, \mathrm{a} \vdash \mathrm{a}}}{! \Sigma \vdash \mathrm{Ax}}(\mathrm{a} \multimap \mathrm{a}) \multimap \mathrm{v} \in \Sigma}{\mathrm{!} \vdash \mathrm{b} \multimap(\mathrm{v} \multimap \mathrm{v}) \in \Sigma} \\
& \text { : applied } n-1 \text { times } \\
& \frac{\left.\frac{1 \Sigma, \mathrm{b} \vdash \mathrm{b}}{\mathrm{A} x}\right\rangle \quad \frac{\mathrm{d}}{! \Sigma, \mathrm{b}^{n-1} \vdash \mathrm{v}}}{\mathrm{l} \Sigma, \mathrm{b}^{n} \vdash \mathrm{v}} \mathrm{b} \multimap(\mathrm{v} \multimap \mathrm{v}) \in \Sigma
\end{aligned}
$$

Lemma 3.11 For any $r, m, n \in \mathbb{N}$ and any $i \in[0, l]$, if $(i, m, n) \rightarrow^{r}(0,0,0)$ then the sequent $! \Sigma, \mathrm{a}^{m}, \mathrm{~b}^{n} \vdash \mathrm{q}_{i}$ is provable in Gill $^{0}$.

Proof. We provide proof trees for the cases that where left to the reader in the body of the paper. Recall that $P$ is a proof tree for the sequent $! \Sigma, \mathrm{a}^{m^{\prime}}, \mathrm{b}^{n^{\prime}} \vdash \mathrm{q}_{i^{\prime}}$.

- Case where $\psi(i)=\left(+, \mathrm{b}, i^{\prime}\right), m^{\prime}=m$ and $n^{\prime}=n+1$. Here is a proof tree for $! \Sigma, \mathrm{a}^{m}, \mathrm{~b}^{n} \vdash \mathrm{q}_{i}$ :

$$
\frac{P}{\frac{! \Sigma, \mathrm{a}^{m}, \mathrm{~b}^{n}, \mathrm{~b} \vdash \mathrm{q}_{i^{\prime}}}{! \Sigma, \mathrm{a}^{m}, \mathrm{~b}^{n} \vdash \mathrm{q}_{i}}\left(\mathrm{~b} \multimap \mathrm{q}_{i^{\prime}}\right) \multimap \mathrm{q}_{i} \in \Sigma}
$$

- Case where $\psi(i)=\left(-, \mathrm{b}, i^{\prime}, k\right), m=m^{\prime}$ and $n=$ $n^{\prime}=0$. Let $Q$ be a proof tree for $! \Sigma, \mathrm{a}^{m} \vdash \mathrm{u}$ according to Proposition 3.10. Here is a proof tree for $! \Sigma, \mathrm{b}^{n} \vdash \mathrm{q}_{i}$ :

$$
\frac{\frac{Q}{! \Sigma, \mathrm{a}^{m} \vdash \mathrm{u}} \frac{P}{! \Sigma, \mathrm{a}^{m} \vdash \mathrm{q}_{i^{\prime}}}}{! \Sigma, \mathrm{a}^{m} \vdash \mathrm{q}_{i}}\left(\mathrm{u} \& \mathrm{q}_{i^{\prime}}\right) \multimap \mathrm{q}_{i} \in \Sigma
$$


- Case where $\psi(i)=\left(-, \mathrm{b}, j, i^{\prime}\right), m^{\prime}=m$ and $n^{\prime}+1=$ $n$. Here is a proof tree for $! \Sigma, \mathrm{a}^{m^{\prime}}, \mathrm{b}, \mathrm{b}^{n^{\prime}} \vdash \mathrm{q}_{i}$ :

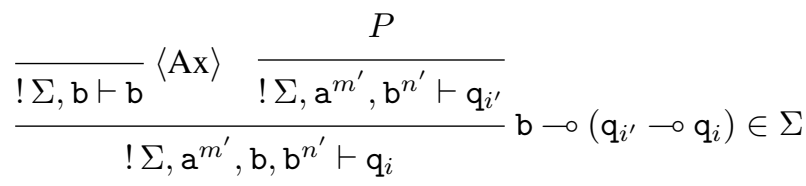

